1

\title{
CFD modelling of particle shrinkage in a fluidized bed for biomass fast
}

\section{pyrolysis with quadrature method of moment}

Bo Liua, Konstantinos Papadikis ${ }^{\mathrm{b}}$, Sai Guc ${ }^{\mathrm{c}}$, Beatriz Fidalgo ${ }^{\mathrm{a}}$, Philip Longhurst ${ }^{\mathrm{a}}$, Zhongyuan $\mathrm{Li}^{\mathrm{a}}$ and

(1)

Athanasios Kolios ${ }^{\mathrm{a}}$

a - Bioenergy and Resource Management Centre, Energy Theme, Cranfield University, College Road, Cranfield, Bedfordshire MK43 0AL, UK;

b - Department of Civil Engineering, Xi'an Jiaotong Liverpool University, 111 Ren'ai Road, Suzhou Dushu Lake Science and Education

Innovation District, Suzhou Industrial Park, Suzhou 215123, China;

c - Department of Chemical \& Process Engineering, University of Surrey, Guildford, Surrey GU2 7XH, UK

\section{Abstract:}

An Eulerian-Eulerian multi-phase CFD model was set up to simulate a lab-scale fluidized bed reactor for the fast pyrolysis of biomass. Biomass particles and the bed material (sand) were considered to be particulate phases and modelled using the kinetic theory of granular flow. A global, multi-stage chemical kinetic mechanism was integrated into the main framework of the CFD model and employed to account for the process of biomass devolatilization. A 3-parameter shrinkage model was used to describe the variation in particle size due to biomass decomposition. This particle shrinkage model was then used in combination with a quadrature method of moment (QMOM) to solve the particle population balance equation (PBE). The evolution of biomass particle size in the fluidized bed was obtained for several different patterns of particle shrinkage, which were represented by different values of shrinkage factors. In addition, pore formation inside the biomass particle was simulated for these shrinkage patterns, and thus, the density variation of biomass particles is taken into account.

\section{Key words:}

Fluidized bed, biomass, fast pyrolysis, CFD, QMOM, particle shrinkage 


\section{Background}

Among the various forms of renewable energy, biomass is becoming a promising resource for energy production, especially transportation fuel, as it has a huge potential for substituting fossil fuels on a large scale. This would relieve the strong dependency of mankind on the petroleum industry and contribute to tackling environmental problems, such as climate change and global warming. Biofuel production is currently based mainly on edible crops, i.e. starch and sugar in the case of bioethanol, and vegetable oils in the case of biodiesel. The use of food crops for the production of $1^{\text {st }}$ generation biofuels may have negative effects on food production, including supply, prices and long term soil depletion [1]. In contrast, lignocellulosic biomass such as energy crops, forestry and agricultural residues, are a lower cost resource as these are not in direct competition with the food supply $[2,3]$. Therefore, advanced bio-fuel technologies based on non-edible feed stocks are more attractive biobased industry options for the future.

Bridgwater $[4,5]$ provides a comprehensive analysis of the thermal conversion of biomass from an economic and technical perspective. Thermal treatment, particularly pyrolysis and gasification, is potentially the most economic conversion process for producing biofuel in competition with oil-based derivatives for storage and use in transportation [6, 7]. Of these methods, fast pyrolysis of lignocellulosic biomass has considerable advantages for producing liquid bio-oil [8]. In the past two decades, intensive research studies on fast pyrolysis have resulted in the design of a series of different reactors such as ablative, auger, entrained flow, vacuum, rotating cone, bubbling fluidized bed and circulating fluidized bed, etc. [9]. Among these developments, fluidized bed reactors have been proven to have a high thermal efficiency and stable product quality as a result of their fast heating and excellent gas-solid mass transfer rate [10].

Biomass fast pyrolysis in a fluidized bed reactor is an extremely complex process as it involves a wide range of chemical and physical phenomena across multiple scales of time and space. Typical examples include descriptions of gas-solid two-phase flow and mixing, turbulent dispersion, mass and heat 
transfer and heterogeneous reactions [11]. Studying such complex processes not only requires the chemical mechanism of pyrolysis to be determined from the molecular level, but importantly coupling this with the gaseous and particulate flow environment. Understanding the behaviour of biomass particles in a fluidized bed is central to determining the product distribution in order to optimize the bio-oil quality [12]. With the rapid development of computing capability, the cutting-edge CFD (Computational Fluid Dynamics) method becomes a good alternative to take the place of traditional experiments in studying the massive flow and decomposition of particles in a fluidized bed reactor. Whilst there are still problems and challenges for multi-phase flow in CFD, especially multi-physics processes, CFD gives acceptable predictions about the hydrodynamic characteristics of the fluidized bed [13-15]. Within the past ten years, effort has been placed on developing comprehensive, computational and predictive CFD models for biomass pyrolysis within fluidized bed reactors. According to differing views on particle dynamics in a CFD framework, existing models can be classified into two basic categories: the Eulerian method and the Lagrangian method. Models in both categories give successful predictions on the general properties of fast pyrolysis in a fluidized bed, such as particle motion, heat transfer and mass transfer, as well as rate of biomass conversion and product yield [16].

The Eulerian method is preferred by many researchers due to its low computing cost, good predictive capability, and relative ease in computer programming. Hence, the Eulerian method has been widely used in previous studies modelling fluidized bed reactors. Lathouwers and Bellan $[17,18]$ provide an example of a comprehensive model based on Eulerian multi-phase fluid dynamics and the kinetic theory of granular flow. They integrated the decomposition mechanism of biomass particles into their CFD model to investigate the effects of operating parameters on product yields in a lab-scale fluidized bed. Gerhauser et al. [19] carried out a more detailed modelling study focusing on the hydrodynamics of the fluidized bed, whereas, Gerber et al. [20] set up an Eulerian-based model to simulate the pyrolysis reactor with char particles as the fluidized medium so as to compare their numerical results with experimental data. Xue et al. [21, 22] and Xue \& Fox [12] developed a CFD model that accounts for variations in biomass particle density caused by devolatilization. Their study assumes a continuous 
loss of mass due to pyrolysis reactions making each particle more porous without changing its size. Mellin et al. $[23,24]$ conducted a 3-D CFD simulation of a lab-scale fluidized bed and included a more detailed prediction of gaseous and liquid product distribution by implementing a comprehensive kinetic model of biomass pyrolysis proposed earlier by Ranzi et al. [25]. In contrast to Eulerian method examples, Fletcher et al.'s [26] work is based on the Lagrangian approach. In this study, the motion of the biomass particle is tracked by applying Newton's law, ignoring particle collision and employing a global reaction mechanism to account for particle decomposition. Papadikis et al. [27-30] proposed a method to simulate a single biomass particle in a pre-fluidized bed based on an Eulerian-EulerianLagrangian CFD framework. The parameters of particle motion, heat transfer between the particle and the bed medium, internal heat conduction and reaction, and particle shrinkage, were systematically studied. Bruchmuller et al. [31] tracked 0.8 million red oak particles inside a pyrolysis fluidized bed reactor with the Lagrangian method and validated their model with experimental results.

Although a number of relatively detailed CFD models which describe the complex gas-solid reactive flow in a bubbling fluidized bed for biomass fast pyrolysis have been developed, none of them completely addresses all the chemical and physical phenomena involved due to the complexity of the system itself. In general, researchers have focused their attention on defining the motion of particles and their transport processes rather than their physical change and therefore the properties of biomass particles within CFD models, e.g. porosity, size, and shape. Despite this, it is known that biomass particles' changing size due to breakage and shrinkage occurs at high frequency during the devolatilization process $[18,32-33,34]$. As a result, interactions between the biomass particle and the bed medium are likely to change at the particle size and density changes. For example, the drag force is directly affected, and consequently, the particle motion is likely to differ from cases where the size and density changes are ignored; spatial distribution and the residence time of char particles in the fluidized bed are also likely to be affected. The implication is that these changes are likely to impact on secondary reaction sequences and the operating status of the reactor. It is this variation in size, especially particle shrinkage, that is one of the most common phenomena of particle change during 
the devolatilization process. The phenomenon of biomass shrinkage during the pyrolysis process has been studied by several researchers [34, 35, 36-37]. The most notable work among these is the 3 parameter shrinkage model proposed by Di Blasi. Despite this progress, the model can only be used to predict the shrinkage of a single biomass particle at a given thermal condition but not the true evolution of particle size in the complex reactor environment. Fan and Fox [13], Fan et al. [38, 39], Marchisio \& Fox [40] and Passalacqua et al. [41] propose a direct quadrature method of moment (DQMOM) and combine this with the Eulerian multi-phase CFD model to describe the process of particle mixing and segregation in a fluidized bed. Xue and Fox [12] further applied this method in their CFD model to predict the distribution of biomass particle sizes in a bubbling fluidized bed during fast pyrolysis. They argue that defining three quadrature abscissas guarantees a high accuracy in determining the continuous particle size distribution; however, particle size variation was not taken into account in this model.

In this paper, the Di Blasi 3-parameter particle shrinkage model is integrated into an Eulerian-based multi-phase CFD framework in order to account for the evolution in particle size throughout the fluidized bed. The quadrature method of moment (QMOM) is employed to solve the particle population balance equation (PBE). This then determines the change in average particle diameter from the biomass devolatilization. Differing shrinkage parameter values are used to represent differing shrinkage patterns. These were investigated to find out how shrinkage affects the particle motion, heating rate and the product yields. For the sake of simplicity, a multi-stage global kinetic model based on pseudo-components was used to account for the chemical conversion of the biomass feed stock. In addition to variation in particle size, the variation in density of the biomass was taken into account. To best of our knowledge, no work similar to this has been reported that studies the size variation for massive particle flow in the fluidized bed reactor during the fast pyrolysis of biomass. 


\section{Mathematical modelling}

124

\subsection{Governing equations}

The basic idea underpinning the Eulerian model as it is used for multi-phase granular flow is to consider each phase, including the physical continuous and discrete phases, as interpenetrative fluids.

Momentum equation of the solid phase is then closured with the kinetic theory in terms of models to calculate the solid viscosity and solid pressure. A detailed description of the multi-phase Eulerian model and kinetic theory can be found elsewhere in the multi-phase flow literature [42]. Table 1 gives a summary of the governing equations used in simulation of a fluidized bed reactor. Solid shear viscosity usually contains three main contributions, i.e. the collision viscosity, kinetic viscosity and frictional viscosity. In this study, collision viscosity is calculated according to Gidaspow et al. [43], whilst kinetic viscosity is accounted for with the correlation of Syamlal et al. [44]. Frictional viscosity is added due to high solid hold up and calculated with the model proposed by Schaeffer [45] using an internal frictional angle of 55 [12]. The radial distribution function $g_{0, s s}$ and the solid pressure $p_{s}$ are calculated according to Lun et al. [46]. The restitution coefficient $e_{s s}$ takes the value of 0.9. The solid granular temperature $\Theta_{s}$ is calculated with the correlation proposed by Syamlal et al. [44]. For multiphase flow problems, momentum interactions between each pair of phases arises due to the drag force, which contributes as a source term $R_{i, j}$ in the phase momentum equations. A widely used drag model proposed by Gidaspow et al. [43] is employed to calculate the gas-solid phase interaction coefficient $K_{i, j}$. The Syamlal-O'Brien-Symmetric model [47] is then used to calculate the drag coefficient between the biomass particles and the sand phase.

Table 1 Governing equations of the fluidized bed reactor based on Eulerian-Granular theory

\section{Models and equations}

\begin{tabular}{ll}
\hline $\begin{array}{l}\text { Continuity } \\
\text { Gas }\end{array}$ & $\frac{\partial \varepsilon_{l} \rho_{l}}{\partial t}+\nabla \cdot\left(\varepsilon_{l} \rho_{l} \mathbf{u}_{1}\right)=\sum_{j=l, j \neq l}^{3}\left(m_{j, l}-m_{l, j}\right)+S_{l}$ \\
Solid (sand/biomass) & $\frac{\partial \varepsilon_{i} \rho_{i}}{\partial t}+\nabla \cdot\left(\varepsilon_{i} \rho_{i} \mathbf{u}_{i}\right)=\sum_{j=1, j \neq i}^{3}\left(m_{j, i}-m_{i, j}\right)+S_{i} \quad i=2,3$
\end{tabular}




\section{Momentum}

Gas

Solid (sand/biomass)

\section{Granular kinetic models}

Solid shear viscosity

Collision viscosity

Kinetic viscosity

Frictional viscosity

Solid bulk viscosity

Radial distribution function

Solid pressure

Granular temperature

Collision dissipation energy

Transfer of kinetic energy

\section{Species transport}

Energy $\frac{\partial \varepsilon_{l} \rho_{l} \mathbf{u}_{1}}{\partial t}+\nabla \cdot\left(\varepsilon_{l} \rho_{l} \mathbf{u}_{l} \mathbf{u}_{l}\right)=-\varepsilon_{l} \nabla p+\nabla \cdot \overline{\bar{\tau}}_{1}+\sum_{j=l, j \neq l}^{3}\left(\mathbf{R}_{l, j}+m_{j, l} \mathbf{u}_{j, l}-m_{l, j} \mathbf{u}_{l, j}\right)+\varepsilon_{l} \rho_{l} g$

$$
\overline{\bar{\tau}}_{1}=\varepsilon_{1} \mu_{l}\left(\nabla \mathbf{u}_{1}+\nabla \mathbf{u}_{l}^{\mathbf{T}}\right)-\frac{2}{3} \varepsilon_{1} \mu_{l} \nabla \cdot \mathbf{u}_{1} \overline{\bar{I}} ; \quad \mathbf{R}_{l, j}=K_{l, j}\left(\mathbf{u}_{j}-\mathbf{u}_{l}\right)
$$$$
\frac{\partial \varepsilon_{i} \rho_{i} \mathbf{u}_{i}}{\partial t}+\nabla \cdot\left(\varepsilon_{i} \rho_{i} \mathbf{u}_{i} \mathbf{u}_{i}\right)=-\varepsilon_{i} \nabla p-\nabla p_{i}+\nabla \cdot \overline{\bar{\tau}}_{i}+\sum_{j=1, j \neq i}^{3}\left(\mathbf{R}_{i, j}+m_{j, i} \mathbf{u}_{j, i}-m_{i, j} \mathbf{u}_{i, j}\right)+\varepsilon_{i} \rho_{i} g
$$

$$
\overline{\bar{\tau}}_{i}=\varepsilon_{i} \mu_{i}\left(\nabla \mathbf{u}_{i}+\nabla \mathbf{u}_{i}^{\mathbf{T}}\right)+\varepsilon_{i}\left(\lambda_{i}-\frac{2}{3} \mu_{i}\right) \nabla \cdot \mathbf{u}_{i} \overline{\bar{I}} \quad i=2,3 ; \quad \mathbf{R}_{i, j}=K_{i, j}\left(\mathbf{u}_{j}-\mathbf{u}_{i}\right)
$$

$$
\mu_{s}=\mu_{s, c o l}+\mu_{s, k i n}+\mu_{s, f r}
$$$$
\mu_{s, c o l}=\frac{4}{5} \varepsilon_{s}^{2} \rho_{s} d_{s} g_{0, s s}\left(1+e_{s s}\right) \sqrt{\frac{\Theta_{s}}{\pi}}
$$$$
\mu_{s, k i n}=\frac{\varepsilon_{s} \rho_{s} d_{s} \sqrt{\Theta} \pi}{6\left(3-e_{s s}\right)}\left[1+0.4\left(1+e_{s s}\right)\left(3 e_{s s}-1\right) \varepsilon_{s} g_{0, s s}\right]
$$

$$
\mu_{s, f r}=\frac{p_{s} \sin \phi}{2 \sqrt{I_{2 D}}}
$$

$$
\lambda_{s}=\frac{4}{3} \varepsilon_{s} \rho_{s} d_{s} g_{0, s s}\left(1+e_{s s}\right) \sqrt{\frac{\Theta_{s}}{\pi}}
$$

$$
g_{0, s s}=\left[1-\left(\frac{\varepsilon_{s}}{\varepsilon_{s, \max }}\right)^{1 / 3}\right]^{-1}
$$

$$
p_{s}=\varepsilon_{s} \rho_{s} \Theta_{s}+2 \rho_{s}\left(1+e_{s s}\right) \varepsilon_{s}^{2} g_{0, s s} \Theta_{s}
$$

$$
\frac{3}{2}\left[\frac{\partial}{\partial t}\left(\rho_{s} \varepsilon_{s} \Theta_{s}\right)+\nabla \cdot\left(\rho_{s} \varepsilon_{s} \Theta_{s}\right)\right]=\left(-p_{s} \overline{\bar{I}}+\bar{\tau}_{s}\right): \nabla \mathbf{u}_{s}+\nabla \cdot\left(k_{\Theta_{s}} \nabla \Theta_{s}\right)-\gamma_{\Theta_{s}}+\varphi_{g, s}
$$

$\gamma_{\Theta_{s}}=\frac{12\left(1-e_{s s}^{2}\right) g_{0, s s}}{d_{s} \sqrt{\pi}} \rho_{s} \varepsilon_{s}^{2} \Theta_{s}^{3 / 2}$

$\varphi_{g, s}=-3 K_{g, s} \Theta_{s}$

$\frac{\partial \varepsilon_{i} \rho_{i} x_{i, p}}{\partial t}+\nabla \cdot\left(\varepsilon_{i} \rho_{i} \mathbf{u}_{i} x_{i, q}\right)=\nabla \cdot\left(\varepsilon_{i} D_{i, q} \nabla x_{i, q}\right)+\mathfrak{R}_{i, q}+\sum_{j=1}^{N}\left(m_{j^{p}, i^{q}}-m_{i^{q}, j^{p}}\right)+\varepsilon_{i} S_{i, q} \quad i=1,3$

$\frac{\partial \varepsilon_{i} \rho_{i} h_{i}}{\partial t}+\nabla \cdot\left(\varepsilon_{i} \mathbf{u}_{i} \rho_{i} h_{i}\right)=\varepsilon_{i} \frac{\partial p}{\partial t}+\overline{\bar{\tau}}_{i}: \nabla \mathbf{u}_{i}+\sum_{j=1, j \neq i}^{3}\left(\mathbf{R}_{i, j} \cdot \mathbf{u}_{i}+Q_{i, j}+m_{j, i} h_{j}-m_{i, j} h_{i}\right)$

$$
+\nabla \cdot\left(\lambda_{i} \nabla T\right)+S_{i}
$$

Note: Phase index i, j: 1 - gas phase; 2 - sand phase; 3 - biomass phase significant energy sources from the fast pyrolysis of biomass in a fluidized bed. Table 2 gives the thermodynamic parameters used in this work. Interphase heat transfer in a biomass fast pyrolysis fluidized bed is extremely complex due to a variety of different physical heat transfer processes occurring simultaneously, such as gas-solid convective heat transfer, solid-solid conductive heat 
transfer and radiative heat transfer. Thus far, no work has been done to account for all these heat transfer processes in a single mathematical model. Most of the existing studies are concerned mainly with the gas-solid heat transfer which is likely to be dominant in particle heating. In this study, the conductive and radiative heat transfer effects of the sand phase are not taken into account. A wellknown Nusselt correlation proposed by Gunn [48] was employed in this work to account for interphase heat transfer between the fluidizing gas and the sand phase. Since the volumetric concentration of the biomass phase is very low throughout the fluidized bed, existence of the biomass particles can be ignored when calculating the gas-sand heat transfer coefficient. Heat transfer between the biomass particles and the bed medium was calculated according to Collier et al. [49], who proposed a modified Nusselt correlation in their studies on heat transfer between a free-moving bronze sphere and the dense fluidizing medium (gas and inert particles). They argue that gas-solid heat transfer is dominant when the heat transfer sphere is smaller than the bed particles, which is exactly the case in the current study.

$$
N u=2+0.9 \operatorname{Re}^{0.62}\left(d_{b} / d_{s}\right)^{0.2}
$$

Where, $d_{b}$ is the diameter of the biomass particle; $d_{s}$ is the diameter of the sand particle.

Table 2 Thermodynamic parameters used in the simulation

\begin{tabular}{lllll}
\hline Species & $C_{p}\left(\mathrm{~kJ}^{\mathrm{kg}} \mathrm{kg}^{-1} \cdot \mathrm{K}^{-1}\right)$ & \multicolumn{2}{l}{$\lambda\left(\mathrm{W} \cdot \mathrm{m}^{-1} \cdot \mathrm{K}^{-1}\right)$} \\
\hline Cellulose, activated cellulose & 2.3 & {$[50]$} & 0.2426 & {$[50]$} \\
Hemicellulose, activated hemicellulose & 2.3 & {$[50]$} & 0.2426 & {$[50]$} \\
Lignin, activated lignin & 2.3 & {$[50]$} & 0.2426 & {$[50]$} \\
Char & 1.1 & {$[50]$} & 0.1046 & {$[51]$} \\
Tar & 2.5 & {$[50]$} & 0.02577 & {$[52]$} \\
Gas, void & 1.1 & {$[51]$} & 0.02577 & {$[51]$} \\
Nitrogen & 1.091 & {$[27]$} & 0.0563 & {$[27]$} \\
Sand & 830 & {$[24]$} & 0.25 & {$[24]$} \\
\hline
\end{tabular}


171 Since this paper focuses mainly on demonstrating a method to describe the particle density

172 and size change in a fluidized bed reactor, rather than accurately predicting product yields, a

173 global chemical kinetic mechanism satisfies the current model in accounting for the biomass

174 devolatilization process. Shafizadeh and Bradbury [53] argue that, in the process of cellulose

175 pyrolysis, an activated intermediate is first produced, then two competitive conversion routes

176 occur afterwards, one which produces condensable bio-oil, and the other which gives

177 permanent gas and char. Ward and Braslaw [54], Koufopanos et al. [55], and Miller and Bellan

178 [56] extend this mechanism to the other main components of lignocellulosic biomass -

179 hemicellulose and lignin, and thus obtain a collective kinetic mechanism for biomass pyrolysis, 180 see Figure 1.

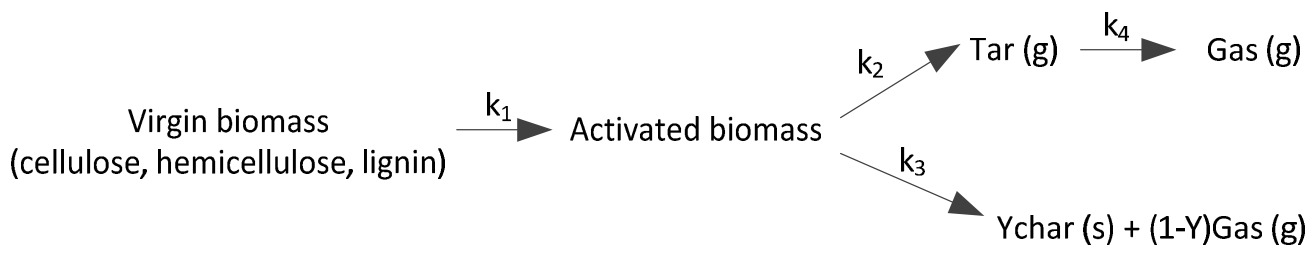

Fig. 1 A multi-stage global reaction mechanism of biomass fast pyrolysis

183 The biomass feedstock used in this work was assumed to be composed of $41 \mathrm{wt} . \%$ cellulose,

$18432 w t . \%$ hemicellulose, and $27 w t . \%$ lignin. This is a typical woody biomass composition [57].

185 Chemical reactions 1-4 from Figure 1 are first-order Arrhenius reactions with respect to the 186 corresponding reactant. The kinetic parameters are shown in Table 3, where $Y$ value in reaction 3 depends on the specific components.

Table 3 Kinetic parameters and reaction heat of biomass pyrolysis

\begin{tabular}{llllllll}
\hline Reaction & $Y$ & $\mathrm{~A}\left(\mathrm{~s}^{-1}\right)$ & $E\left(\mathrm{~kJ} \cdot \mathrm{mol}^{-1}\right)$ & \multicolumn{2}{c}{$k_{T=773 \mathrm{~K}}\left(\mathrm{~kg} \cdot \mathrm{m}^{-3} \cdot \mathrm{s}^{-1}\right)$} & $\Delta h$ & $\left(\mathrm{~kJ} \cdot \mathrm{kg}^{-1}\right)$ \\
\cline { 2 - 7 }$k_{1, c}$ & $2.8 \times 10^{19}$ & 242.4 & 539.246 & {$[53]$} & 0 & {$[50]$} \\
$k_{2, c}$ & & $3.28 \times 10^{14}$ & 196.5 & 8.775 & {$[53]$} & 255 & {$[58]$} \\
$k_{3, c}$ & 0.35 & $1.3 \times 10^{10}$ & 150.5 & 0.5232 & {$[53]$} & -20 & {$[58]$} \\
$k_{1, h}$ & & $2.1 \times 10^{16}$ & 186.7 & 2670.041 & {$[54]$} & 0 & {$[58]$} \\
$k_{2, h}$ & & $8.75 \times 10^{15}$ & 202.4 & 91.597 & {$[56]$} & 255 & {$[58]$} \\
\hline
\end{tabular}




\begin{tabular}{llllllll}
\hline$k_{3, h}$ & 0.6 & $2.6 \times 10^{11}$ & 145.7 & 22.450 & {$[56]$} & -20 & {$[58]$} \\
$k_{1, l}$ & & $9.6 \times 10^{8}$ & 107.6 & 35.493 & {$[54]$} & 0 & {$[58]$} \\
$k_{2,1}$ & & $1.5 \times 10^{9}$ & 143.8 & 0.175 & {$[56]$} & 255 & {$[58]$} \\
$k_{3,1}$ & 0.75 & $7.7 \times 10^{6}$ & 111.4 & 0.156 & {$[56]$} & -20 & {$[58]$} \\
$k_{4}$ & & $4.28 \times 10^{6}$ & 108.0 & 0.147 & {$[59]$} & -42 & {$[50]$} \\
\hline
\end{tabular}

Note: subscript $c$ represents cellulose; $h$ represents hemicellulose; / represents lignin.

207 fraction can be derived directly from the law of unity: $x_{i, n}=1-\sum_{k=1}^{n-1} x_{i, k}$. constitute the particle.

$$
\rho_{\text {apparent }}=\left(\sum_{q=1}^{N} \frac{x_{q}}{\rho_{q}}\right)^{-1}
$$

The continuous loss of particle mass from devolatilization makes the biomass particles shrink and become more porous. Pore formation plays an important role in the apparent density change of the biomass particle. It is assumed that pores which form inside the biomass particle fill with permanent gas produced by the decomposition process. In other words, the majority of permanent gas produced is released to the gas phase, and a small fraction remains in the biomass particle, forming pores. Indeed, biomass particles maintain a very small holdup within the fluidized bed [21, 23], so the total amount of permanent gas inside the pores is extremely small and can be ignored when compared with the counterpart released to the gas phase. Therefore, this assumption incurs no significant artificial errors and provides a simple way to account for the apparent density change. The apparent density of the biomass particle is defined as the volume-weighted-average density of the component true densities, including virgin biomass, activated biomass, char, and permanent gas in pores that

Where, $x_{q}$ is the mass fraction of the $q^{\text {th }}$ component in the biomass phase, which has exactly the same meaning as $x_{3, q}$ in the species equation; $\rho_{q}$ is the true density of the $q^{\text {th }}$ component. All phases and species involved in the current CFD model were numbered as shown in Table 4. Only $n-1$ transport equations were actually solved for each phase which contains $n$ species in all. The $n^{\text {th }}$ component mass

Table 4 Reference number of species in gas and biomass phase

\begin{tabular}{lcc}
\hline Phases and components & Phase No. & Component No. in each phase \\
\hline Gas phase & 1 & 1 \\
Tar & & 2 \\
Gas & & 3 \\
$\mathrm{~N}_{2}$ & 2 & - \\
Sand phase & & \\
$\quad$ Sand & & \\
\hline
\end{tabular}




\begin{tabular}{ll}
\hline Biomass phase & 3 \\
$\quad$ Char & 1 \\
Activated-lignin & 2 \\
Activated-hemicellulose & 3 \\
Activated-cellulose & 4 \\
Lignin & 5 \\
Hemicellulose & 6 \\
Cellulose & 7 \\
Void & 8 \\
Ash & 9 \\
\hline
\end{tabular}
the reaction source term $R_{i, q}$ for each species can be written as follows.

$$
\begin{aligned}
& \mathfrak{R}_{1,1}=\rho_{3} \varepsilon_{3} k_{2, c} x_{3,4}+\rho_{3} \varepsilon_{3} k_{2, h} x_{3,3}+\rho_{3} \varepsilon_{3} k_{2, l} x_{3,2}-\rho_{1} \varepsilon_{l} k_{4} x_{1,1} \\
& \mathfrak{R}_{1,2}=\rho_{3} \varepsilon_{3} k_{3, c}\left(1-Y_{c}\right) x_{3,4}+\rho_{3} \varepsilon_{3} k_{3, h}\left(1-Y_{h}\right) x_{3,3}+\rho_{3} \varepsilon_{3} k_{3, l}\left(1-Y_{l}\right) x_{3,2}+\rho_{1} \varepsilon_{l} k_{4} x_{1,1} \\
& \mathfrak{R}_{3,1}=\rho_{3} \varepsilon_{3} k_{3, c} Y_{c} x_{3,4}+\rho_{3} \varepsilon_{3} k_{3, h} Y_{h} x_{3,3}+\rho_{3} \varepsilon_{3} k_{3, l} Y_{l} x_{3,2} \\
& \mathfrak{R}_{3,2}=\rho_{3} \varepsilon_{3} k_{1, c} x_{3,5}-\rho_{3} \varepsilon_{3} k_{2, c} x_{3,2} \\
& \mathfrak{R}_{3,3}=\rho_{3} \varepsilon_{3} k_{1, h} x_{3,6}-\rho_{3} \varepsilon_{3} k_{2, c} x_{3,3} \\
& \mathfrak{R}_{3,4}=\rho_{3} \varepsilon_{3} k_{1, c} x_{3,7}-\rho_{3} \varepsilon_{3} k_{2, c} x_{3,4} \\
& \mathfrak{R}_{3,5}=-\rho_{3} \varepsilon_{3} k_{1, l} x_{3,5} \\
& \mathfrak{R}_{3,6}=-\rho_{3} \varepsilon_{3} k_{1, h} x_{3,6} \\
& \mathfrak{R}_{3,7}=-\rho_{3} \varepsilon_{3} k_{1, c} x_{3,7}
\end{aligned}
$$

It can be noted in Table 5 that none of the above expressions account for the mass fraction of the component "void" which represents the quantity of pores formed in a devolatilizing biomass particle. Only $R_{1,2}$ gives the total amount of permanent gas produced per unit volume per unit time, in place of an exact distribution ratio for that released to the gas phase and that remaining in the solid particle. Hence, an artificial interphase mass transfer term should be defined to account for the pore formation rate. In the process of biomass fast pyrolysis, the pore formation rate depends on the rate at which the biomass solid disappears because of the occurrence of heterogeneous reactions. This can be affected by the particle shrinking rate as biomass decomposition causes not only pore-formation 
inside a particle but also size change of the particle itself. Disappearance of the solid component in a biomass particle is an overall result of decomposition reactions 2 and 3 . This can be calculated from the chemical kinetics, whilst calculation of the particle shrinking rate needs additional models. In this work the 3-parameter shrinkage model proposed by Di Blasi [34] is used to account for the size change of biomass particles during fast pyrolysis, described in detail in Section 2.4. (PBE)

Population distribution of a particulate system can be described generally with a particle number density function with respect to different particle properties called the "internal coordinates", which include particle size, shape and any other properties distinguishing the particles from one another. In a fluidized bed, the number density is not only a function of these "internal coordinates", but also of "external coordinates", including their spatial position and time. In order to integrate the particle number density function to the current Eulerian multi-phase CFD framework, the conservation law for a particle number with a specific property was applied to each control volume in the computational domain. Since the only internal coordinate concerned in this study is the particle size, the PBE in an Eulerian multi-phase flow framework can be written as in equation (3):

$$
\frac{\partial n(L)}{\partial t}+\nabla \cdot \mathbf{u} n(L)=-\frac{\partial}{\partial L}\left[\frac{\partial L}{\partial t} n(L)\right]
$$

Where, $n(L)$ is the number density function with respect to the particle size $L$. It can be seen clearly that the above equation is a transient Eulerian equation with source terms. The term on the right hand side of equation (3) denotes the source due to particle growth/shrinkage. In general, this equation should include other source terms with respect to particle aggregation and fragmentation. Particle aggregation hardly exists in a biomass fast pyrolysis fluidized bed; however, particle fragmentation does occur. As far as we know, there have been no experimental methods developed to measure the dynamics of particle fragmentation effectively and accurately in this kind of fluidized bed reactor. 
Hence, only the particle shrinkage have been taken into account and other particle processes have been ignored in this work.

Solving equation (3) directly would be extremely time-consuming due to its additional dimension of particle size distribution. Randolph and Larson [60] proposed an indirect method for PBE time evolution by calculating several low order moments of the number density function to reduce the dimensionality and then solve a set of moment conservation equations. Nevertheless, a closure equation set cannot be derived without knowing the particle size distribution. Where complex particle phenomena are taken into account, such as size-dependent growth, particle fragmentation and aggregation, this is especially the case. McGraw [61] approximated these moments with the $n$-point Gaussian quadrature and finally improved the closure of this method for a broader range of particle events. Xue and Fox [12] claimed that three integral quadrature abscissas can produce acceptable simulation results for the particle size evolution in a biomass fast pyrolysis fluidized bed, which means that only the first six, low order moment conservation equations need to be solved. Hence, the PBE equation (3) is replaced by the following moment conservation equation in this study.

$$
\frac{\partial \rho_{s} m_{k}}{\partial t}+\nabla \cdot\left(\rho_{s} \mathbf{u} m_{k}\right)=m_{k} \frac{\partial \rho_{s}}{\partial t}+\mathbf{u} m_{k} \cdot \nabla \rho_{s}-\rho_{s} \int_{0}^{\infty} k d_{s}^{k-1}\left[\frac{\partial d_{s}}{\partial t} n\left(d_{s}\right)\right] d\left(d_{s}\right) \quad k=0, \ldots, 5
$$

Where, $m_{k}$ denotes the moment of $k^{\text {th }}$ order; $n\left(d_{s}\right)$ is the number density function with respect to particle diameter, which has the same meaning as $n(L)$ in equation (3). The zero order moment $m_{0}$ represents the total particle number density, the second order moment $m_{2}$ is related to total particle area, and the third order moment $m_{3}$ relates to the total particle volume. However, based on the definition of the moment, other low-order moments such as $m_{1}, m_{4}$ and $m_{5}$ have no clear physical meanings. 
shrinkage of the char layer formed in a wood slap pyrolysis subjected to a radiation heat flux. The total volume of a biomass particle was considered to be a sum of the pore volume occupied by a gaseous substance plus the solid volume remaining (volume occupied by char, unreacted biomass, and partlyreacted biomass). Therefore, a shrinkage model with three shrinkage factors was proposed, with $\alpha, b$, $\gamma$ representing different shrinkage contributions.

The volume occupied by the solid was assumed to decrease linearly with the biomass components and to increase linearly with the char mass, as devolatilization takes place. The shrinkage factor $\alpha$ reflects the density change of the solid residuals due to char formation, a value of $\alpha$ between 0 and 1 represents no density increase and maximum density increase, respectively.

$$
\frac{V_{S}}{V_{W 0}}=\frac{M_{W}}{M_{W 0}}+\frac{\alpha M_{C}}{M_{W 0}}
$$

Where, $V_{s}$ is the current solid volume; $V_{w o}$ is the initial solid volume; $M_{w}$ is the current mass of the remaining biomass solid; $M_{w o}$ is the initial mass of the biomass solid; $M_{C}$ is the current char mass. The volume occupied by volatiles is composed of two contributions, the first due to the initial volume of pores, $V_{g o}$, and the second due to a fraction $B$ of the volume left by the biomass solid as a consequence of devolatilization, $V_{w o}-V_{s}$.

$$
V_{g}=V_{g 0}+\beta\left(V_{W 0}-V_{S}\right)
$$

The initial volume of volatiles in a biomass particle may also decrease with the size change of the particle, depending on a reaction progress factor $\eta, \eta=M_{w} / M_{w o}$, and shrinkage factor $\gamma$.

$$
V_{g 0}=\eta V_{g i}+(1-\eta) \gamma V_{g i}
$$

Where, $V_{g i}$ is the initial value of $V_{g o}$ before the devolatilization process happens, depending totally on the initial porosity of the biomass particle. The initial porosity of the biomass was assumed to be 0.5 in the current work [62]. 


\subsection{Integration of the particle shrinkage into the CFD model}

295
The key point of introducing the Di Blasi 3-parameter particle shrinkage model into the current CFD framework is to translate the particle shrinkage pattern represented by different shrinkage factors into the apparent density calculation and volume evolution occupied by volatiles inside the biomass particle. The apparent density of the biomass particle is defined as the volume-weighted average of the components' true densities according to equation (2). This can be considered further as the volume-weighted average of the volatile (gaseous substances occupying the void) true density and the solid (unreacted biomass and char) true density. The density of the volatile in pores is assumed to be the same as that of the permanent gas in the gas phase, while the solid density depends on the value of the shrinkage factor $\alpha$ in a specific shrinkage pattern. The volume-weighted mixing law is applied to calculate the density of the remained solid substances and make it consistent with the value of $\alpha$ in a specific shrinkage pattern by assigning a proper value to the char density.

In fact, differing $b$ and $v$ values account for differing manners of volume evolution of the component "void" in the biomass phase. By prescribing a set of these values, the mass transfer term between "void" and permanent gas $m_{1^{2}, 3^{8}}$ can be defined, and then the species transport equations are finally closed. The shrinking rate of the biomass particle is the sum of the shrinking effects contributed from "void" and solid. Equation (6) gives a simple expression that the total particle volume shrinkage rate $R$ (volumetric decreasing rate of the biomass phase per unit volume of the flow domain) is a sum of those corresponding to void and solid volume change, respectively.

$$
R=R_{g}+R_{s}
$$

The volume occupied by the biomass phase in an Eulerian control volume can be calculated with $V_{b}=\varepsilon_{b} V$, where $V$ is the total volume of the control volume. The shrinking rate can then be written as follows:

$$
\frac{d V_{b}}{d t}=-R V
$$


Let both sides of the above equation be divided by $V_{b}$ :

$$
\frac{1}{V_{b}} \frac{d V_{b}}{d t}=-\frac{R V}{V_{b}}
$$

Substituting $V_{b}=N \pi d_{b}^{3} / 6$ into equation (10) results into:

$$
\frac{d\left(d_{b}\right)}{d t}=-\frac{R d_{b}}{3 \varepsilon_{b}}
$$

Equation (11) gives the size-dependent particle shrinking rate, which is exactly the source term as it appears in the moment conservation equations. Three different shrinkage patterns were investigated in the current study, which were related to three sets of shrinkage factors, respectively (See Table 6). different, depending on the selected shrinkage pattern.

$$
\begin{aligned}
& \text { No shrinkage: } \\
& \alpha=1,6=1 \text { and } \gamma=1 \\
& \rho_{\text {char }}=\frac{1}{\alpha} \rho_{b}=\rho_{b} \\
& m_{l^{2}, 3^{8}}=\left[\rho_{3} \varepsilon_{3}\left(k_{2 c}+k_{3 c}\right) x_{3,6}+\rho_{3} \varepsilon_{3}\left(k_{2 h}+k_{3 h}\right) x_{3,7}+\rho_{3} \varepsilon_{3}\left(k_{2 l}+k_{3 l}\right) x_{3,8}\right] \frac{\rho_{\text {void }}}{\rho_{\text {biomass }}} \\
& -\left(\rho_{3} \varepsilon_{3} k_{3 c} Y_{c} x_{3,6}+\rho_{3} \varepsilon_{3} k_{3 h} Y_{h} x_{3,7}+\rho_{3} \varepsilon_{3} k_{3 l} Y_{l} x_{3,8}\right) \frac{\rho_{\text {void }}}{\rho_{\text {char }}} \\
& R_{s}=R_{g}=0 \\
& \text { Shrinkage pattern 1: } \quad \alpha=1,6=0 \text { and } \gamma=1 \\
& \rho_{\text {char }}=\frac{1}{\alpha} \rho_{b}=\rho_{b} \\
& m_{1^{2}, 3^{8}}=0 \\
& R_{g}=0 \\
& R_{s}=\left[\rho_{3} \varepsilon_{3}\left(k_{2 c}+k_{3 c}\right) x_{3,6}+\rho_{3} \varepsilon_{3}\left(k_{2 h}+k_{3 h}\right) x_{3,7}+\rho_{3} \varepsilon_{3}\left(k_{2 l}+k_{3 l}\right) x_{3,8}\right] \frac{1}{\rho_{\text {biomass }}} \\
& -\left(\rho_{3} \varepsilon_{3} k_{3 c} Y_{c} x_{3,6}+\rho_{3} \varepsilon_{3} k_{3 h} Y_{h} x_{3,7}+\rho_{3} \varepsilon_{3} k_{3 l} Y_{l} x_{3,8}\right) \frac{1}{\rho_{\text {char }}} \\
& \rho_{\text {char }}=\frac{1}{\alpha} \rho_{b}=2 \rho_{b} \\
& R_{g}=-m_{1^{2}, 3^{8}}=0.5\left[\rho_{3} \varepsilon_{3}\left(k_{2 c}+k_{3 c}\right) x_{3,6}+\rho_{3} \varepsilon_{3}\left(k_{2 h}+k_{3 h}\right) x_{3,7}+\rho_{3} \varepsilon_{3}\left(k_{2 l}+k_{3 l}\right) x_{3,8}\right] \frac{\rho_{\text {void }}}{\rho_{\text {biomass }}}
\end{aligned}
$$




$$
\begin{aligned}
R_{s} & =\left[\rho_{3} \varepsilon_{3}\left(k_{2 c}+k_{3 c}\right) x_{3,6}+\rho_{3} \varepsilon_{3}\left(k_{2 h}+k_{3 h}\right) x_{3,7}+\rho_{3} \varepsilon_{3}\left(k_{2 l}+k_{3 l}\right) x_{3,8}\right] \frac{1}{\rho_{\text {biomass }}} \\
& -\left(\rho_{3} \varepsilon_{3} k_{3 c} Y_{c} x_{3,6}+\rho_{3} \varepsilon_{3} k_{3 h} Y_{h} x_{3,7}+\rho_{3} \varepsilon_{3} k_{3 l} Y_{l} x_{3,8}\right) \frac{1}{\rho_{\text {char }}}
\end{aligned}
$$

In the case of the no shrinkage pattern, each shrinkage factor takes the value of unity. An artificial mass transfer from the permanent gas to the "void" is required to compensate for the volume loss due to biomass decomposition so that the particle size can remain constant. In the case of shrinkage pattern 1 , shrinkage factor 6 comes to 0 , and the other two factors stay the same as the above case. This means that the "void" volume depends on the initial volume only, i.e. no artificial mass transfer is needed. Particle size change, in this case, is attributed wholly to the net volume loss of the solid substance. In the case of shrinkage pattern 2 , the initial volume of the volatile varies with the reaction progress. It is assumed that $50 \%$ of the initial volatiles leave the biomass particle as a consequence of particle size decrease. So, an artificial mass transfer from the "void" to permanent gas needs to be defined to account for this gaseous volume loss. It should be noted that, though the artificial mass transfers introduced in the case of no shrinkage pattern and shrinkage pattern 2 are both between permanent gas and the "void", the mass transfer directions are the opposite.

\section{Model setups and solution strategy}

\subsection{Model geometry and solution strategy}

The geometrical model of the simulation in this study is based on a $300 \mathrm{~g} / \mathrm{h}$ lab-scale cylinder fluidized bed reactor for biomass fast pyrolysis. Simulation was carried out using a $40 \mathrm{~mm} \times 340 \mathrm{~mm} 2-\mathrm{D}$ computational domain, shown in Figure 2, and considering a height of the pre-loaded sand of $80 \mathrm{~mm}$. 


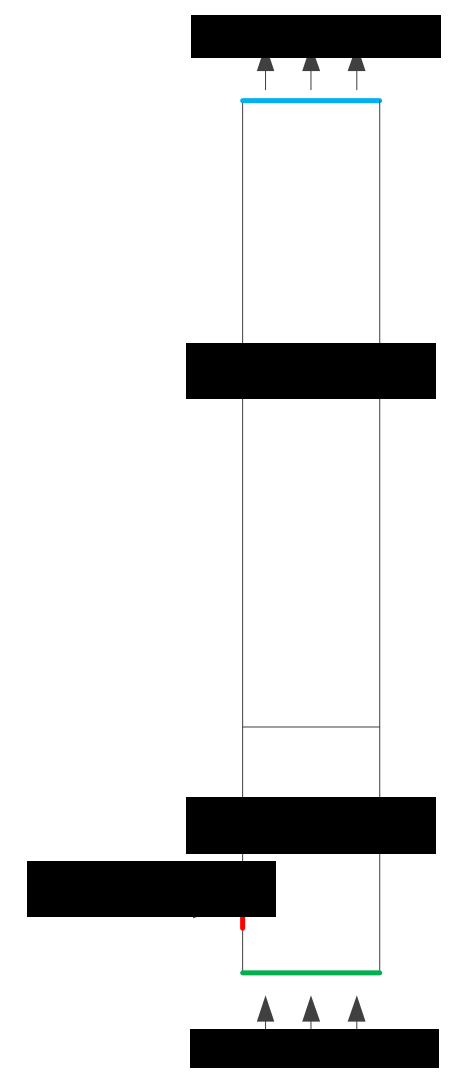

Fig. 2 The 2-D computational domain of the fluidized bed for numerical simulation

The phase-coupled SIMPLE algorithm was employed for pressure-velocity coupling. A volume of fraction (VOF) equation was obtained for each solid phase from the total mass continuity. An explicit variable arrangement was used for the VOF equation. Updating of the VOF at each iteration, was included to achieve timely and better convergence, with a guarantee that the volume fraction of each biomass phase matched the density updating. The volume fraction of the gas phase is obtained directly from the law of unity. A second order upwind scheme was generally used in accounting for the convection term discretization in the flow equation, energy equation, and species transport equations. The three order upwind-like QUICK scheme was used for the VOF equations in order to obtain high VOF precisions for each secondary phase. For temporal discretization, the first order implicit method was employed. A relatively small time step length of $5 \times 10^{-5} \mathrm{~s}$ was used at the beginning of the simulation to overcome the difficulty of convergence due to poor initial fields, and a fixed time step length of $2 \times 10^{-4} \mathrm{~s}$ was used afterwards when simulation became stable. 
361 The biomass particles were assumed to be perfect spheres with a Sauter mean diameter of $325 \mu \mathrm{m}$,

362 following a normal distribution for the particle size. Based on this size distribution, the low order moments of the biomass feedstock were calculated, providing the boundary condition of the PBE at the biomass inlet. Table 7 gives the $0^{\text {th }}-5^{\text {th }}$ order moments of the size distribution.

Table 7 Low order moments of the feed stock

\begin{tabular}{ll}
\hline Moments & Calculation method \\
\hline Moment-0 & $m_{0}=N$ \\
Moment-1 & $m_{1}=\bar{d} m_{0}$ \\
Moment-2 & $m_{2}=2 \bar{d} m_{1}-\bar{d}^{2} m_{0}+\sigma^{2} N^{2}$ \\
Moment-3 & $m_{3}=3 \bar{d} m_{2}-3 \bar{d}^{2} m_{1}+\bar{d}^{3} m_{0}$ \\
Moment-4 & $m_{4}=4 \bar{d} m_{3}-6 \bar{d}^{2} m_{2}+4 \bar{d}^{3} m_{1}-\bar{d}^{4} m_{0}+3 \sigma^{4} N^{4}$ \\
Moment-5 & $m_{5}=\bar{d} m_{4}-10 \bar{d}^{2} m_{3}+10 \bar{d}^{3} m_{2}-5 \bar{d}^{4} m_{1}+\bar{d}^{5} m_{0}$ \\
\hline
\end{tabular}
parameters.

Table 8 Model parameters and simulations conditions

\begin{tabular}{ll}
\hline Parameters & Value \\
\hline Biomass feeding rate $(\mathrm{g} / \mathrm{h})$ & 180 (Equivalent to a cylinder experimental rig ) \\
Nitrogen inflow velocity $(\mathrm{m} / \mathrm{s})$ & 0.3 \\
Minimum fluidized velocity $(\mathrm{m} / \mathrm{s})$ & 0.081 \\
Biomass feeding temperature $(\mathrm{K})$ & 300 \\
Nitrogen feeding temperature $(\mathrm{K})$ & 773 \\
Wall temperature $(\mathrm{K})$ & 773 \\
Gas density $\left(\mathrm{kg} / \mathrm{m}^{3}\right)$ & Incompressible ideal gas law \\
Gas viscosity $(\mathrm{Pa} \cdot \mathrm{s})$ & $3.507 \times 10^{-5}(773 \mathrm{~K})$ \\
Biomass particle size $(\mu \mathrm{m})$ & $325($ Sauter mean) \\
Initial moments of the biomass particle & \\
Moment0 & $6.924 \times 10^{10}$ \\
Moment1 & $1.932 \times 10^{7}$ \\
Moment2 & 5875 \\
Moment3 & 1.910 \\
Moment4 & $6.563 \times 10^{-4}$ \\
Moment5 & $2.366 \times 10^{-7}$ \\
Biomass component true density $\left(\mathrm{kg} / \mathrm{m}^{3}\right)$ & \\
Cellulose, hemicellulose, lignin & 800 \\
Char density for no shrinkage & 800 \\
Char density for shrink pattern 1 & 800 \\
Char density for shrink pattern 2 & 1600 \\
void & Same as gas \\
\hline
\end{tabular}


Biomass component initial mass fraction

$\begin{array}{ll}\text { Cellulose } & 4.094 \times 10^{-1} \\ \text { Hemicellulose } & 3.195 \times 10^{-1} \\ \text { Lignin } & 2.696 \times 10^{-1} \\ \text { void } & 1.529 \times 10^{-3} \\ \text { Biomass initial porosity }\left(\mathrm{m}^{3} \mathrm{gas} / \mathrm{m}^{3} \text { particle) }\right. & 0.5 \\ \text { Biomass initial apparent density }\left(\mathrm{kg} / \mathrm{m}^{3}\right) & 400 \\ \text { Sand particle size }(\mu \mathrm{m}) & 440 \\ \text { Sand density }\left(\mathrm{kg} / \mathrm{m}^{3}\right) & 2500 \\ \text { Sand initial packing height }(\mathrm{mm}) & 80 \\ \text { Sand initial packing limit } & 0.63\end{array}$

\subsection{Implementation of the simulation}

In order to implement the simulation of the mathematical model depicted above, two additional assumptions should be made. First, an even temperature distribution inside the biomass particle is always achieved throughout the simulation; which means internal heat resistance is ignored. The assumption is safe because of the low Bi number of the particle itself. Second, volatiles produced due to the devolatization process are released instantaneously into the gas phase; which means heterogeneous reactions play a dominant part in the interphase mass transfer process instead of diffusion. This assumption is also safe because of the fairly small size of the biomass particle used in this study.

The mathematical model of the fluidized bed was simulated with a widely used CFD package FLUENT 16.2. The interphase mass transfer between "void" and permanent gas, heat transfer between the biomass phase and the bed medium, and source terms of the moment conservation equation due to particle density and size variation, were accounted for by proper DEFINE MACROs of the FLUENT UDF system. Each case in this study was run in parallel on an HPC cluster with 80 computing nodes, each of which has two Intel E5-2660 CPUs, giving 16 CPU cores. Results gained from the simulation are discussed in detail in the following sections. 


\subsection{Operational steady state of the fluidized bed}

Since a mathematically rigorous steady state, in which all the field variables remain constants cannot be reached in a fluidized bed, a transient solver is employed to carry out this kind of simulation. The fluidized bed reactor reaches an operational steady state when the main field parameters reach fixed values statistically at a specific time after the start-up. Not all of the field variables reach steady state simultaneously for a single simulation process. Within this study, the hydrodynamics of the fluidized bed seemed less sensitive to the initial field and achieved a steady state after a few seconds from the beginning of the simulation. Typically, the biomass hold-up of the fluidized bed increased rapidly in the first 5 seconds due to injection and reached an approximate constant value after 20 seconds, when a steady output of the solid residue was set up. In contrast, the thermal steady state depends largely on the initial temperature field. Xue et al. [21] observed that more than 100 seconds were required to reach a thermal steady state when the initial temperature field deviated significantly from the final temperature field, whereas if an appropriate initial temperature field is adopted, a thermal steady state can be achieved in a few seconds. In this work, an initial temperature of $773 \mathrm{~K}$ was used throughout the fluidized bed to guarantee a rapid achievement of the thermal steady state.

\subsection{Grid dependency test}

According to Min et al. [63], numerical simulations with a $<4 \mathrm{~mm}$ mesh can produce predictive results of the main characteristics of a lab-scale fluidized bed. At the beginning of this work, a grid dependency test were carried out with grid resolutions of $4 \mathrm{~mm}, 2 \mathrm{~mm}$ and $1 \mathrm{~mm}$. Results of some most interested parameters of this study such as particle apparent density, particle diameter are plotted against vertical axis of the fluidized bed reactor in Figure 3. Our test shows that all of the three grid resolutions give very close results, especially for outlet values, and can be referred approximately as gridindependent solutions. Additionally, the $1 \mathrm{~mm}$ and the $2 \mathrm{~mm}$ grids seem to give better predictions than the $4 \mathrm{~mm}$ grid across the splashing zone of the fluidized bed, especially for particle size evolution, see 

finally adopted in this study.

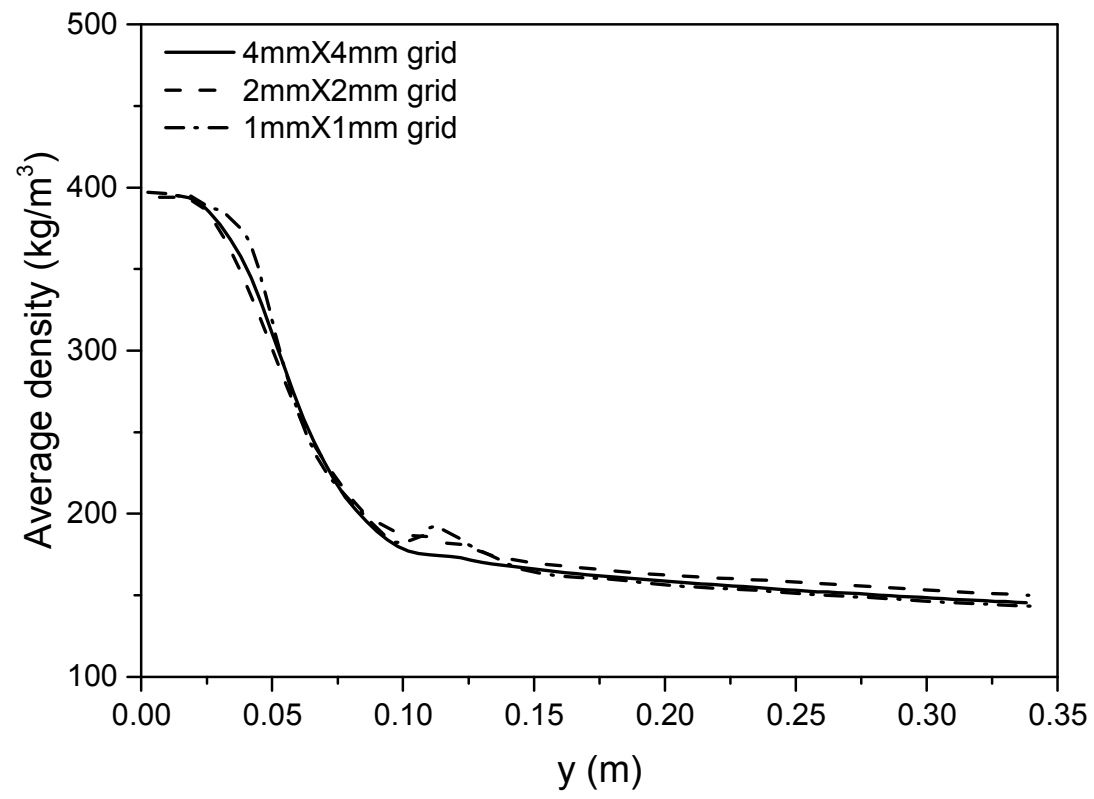

(a)

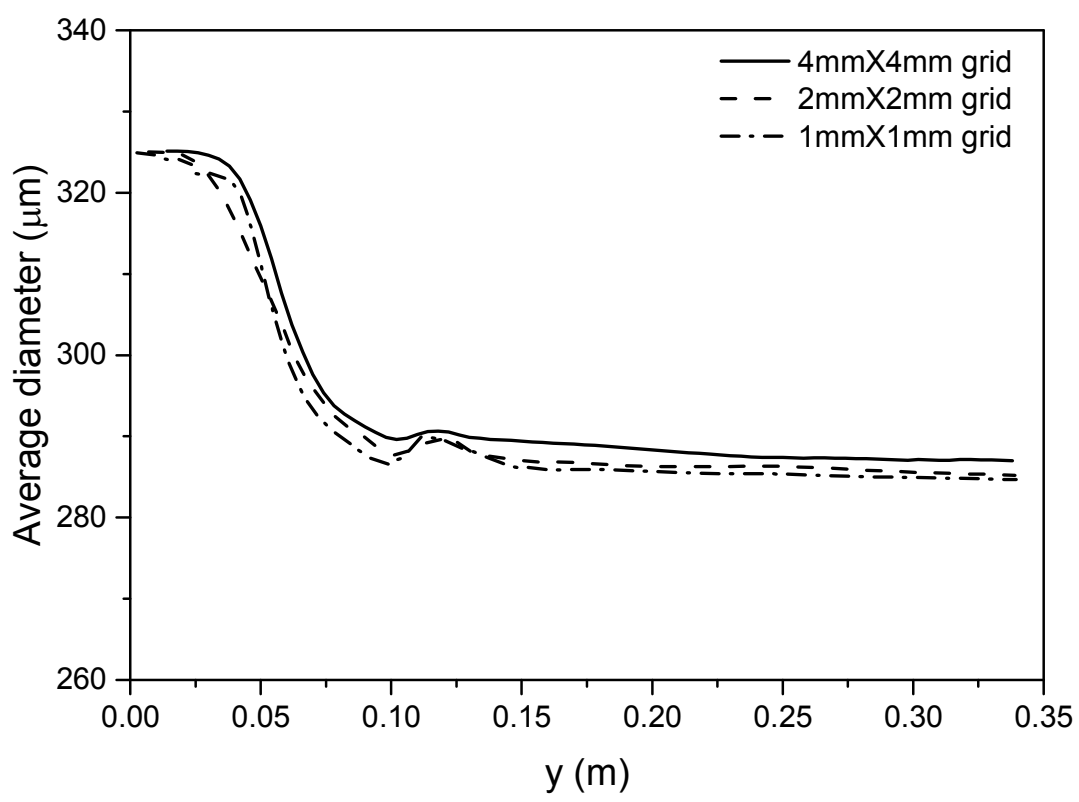

(b)

Fig. 3 Grid dependency test: (a) average density and (b) average particle size of the biomass phase variation along the $y$ axis at steady state (shrinkage pattern 1 )

\subsection{Density and size evolution of the biomass particles}

Figure 4 (a) exhibits snapshots of the gas volume fraction distribution of the fluidized bed reactor corresponding to $0.05 \mathrm{~s}$ at steady state around $24 \mathrm{~s}$ and the particle shrinkage pattern 1 . Results show 
424 typical bubble formation, growth, rise, and breakage within the fluidized bed reactor. Solid particles 425 are firstly lifted up by the rising bubbles and then fall down due to density difference. These chaotic 426 motions of gas and solid particles largely enhance phase mixing, and heat and mass transfers. Cold 427 biomass particles are injected into the reactor and mix rapidly with the hot bed medium. As a result, 428 the volume fraction decreases by almost two orders of magnitude compared to the feeding state. This occurrence can be observed in Figure 4 (b), which shows a snapshot of biomass volume fraction distribution at $24 \mathrm{~s}$ in the case of particle shrinkage pattern 1 . The biomass phase cannot fill the whole dense zone before entering the free board due to short particle residence time, which explains the high concentration region adjacent to the feeding port. Because of the endothermic nature of the decomposition reaction and low temperature of the feedstock (300K), an apparent temperature gradient arises near the high concentration zone for the biomass phase (see Figure 5 (a)). Devolatilization occurs inside the biomass particle when a certain temperature is reached. Pores are formed as a consequence of continuous mass loss due to volatile release, which may result in a drop

437 in apparent density of the biomass particle. On the other hand, apparent density may increase due to the increase in true densities of the solid components according to Di Blasi's shrinkage theory, in which the shrinkage factor $\alpha$ accounts mostly for true density variation of the solid with respect to solid matrix reconstitution happening during the char formation process. 



$24.10 \mathrm{~s}$

Fig. 4 Snapshots of volume fraction distribution at steady state, shrinkage pattern 1: (a) Gas phase; (b) biomass phase
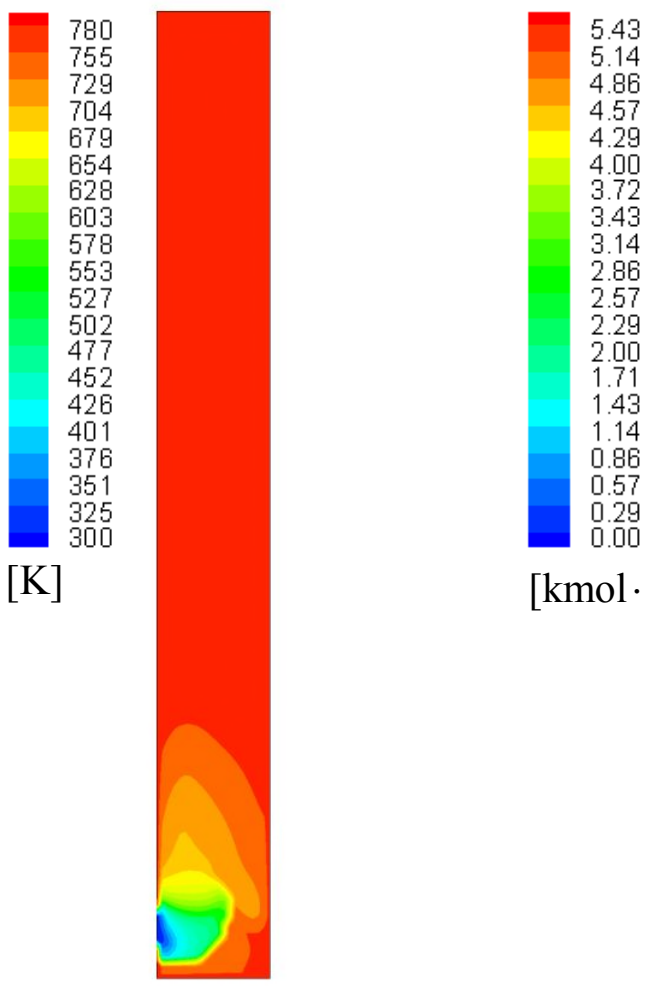

$\left[\mathrm{kmol} \cdot \mathrm{m}^{-3} \cdot \mathrm{s}^{-1}\right]$

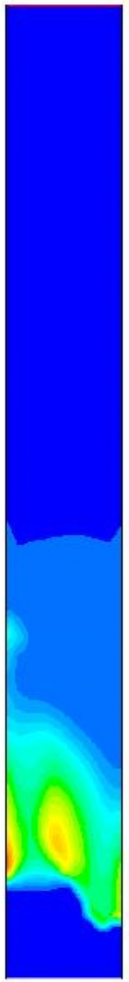

(b) Decomposition rate

Fig. 5 (a) Distribution of temperature and (b) decomposition rate of the biomass phase in the fluidized bed at 24 s (data derived from shrinking pattern 1) 
The apparent density distribution of the biomass phase in a fluidized bed is an overall result of particle motion, heat transfer and heterogeneous reactions. Figure 6 shows the apparent density distribution for biomass particles with different shrinking patterns at 24s. It can be observed that the particle apparent density decreases throughout the vertical axis of the fluidized bed reactor in all of the three shrinkage patterns. A sharp density gradient is observed close to the biomass injection point in the dense zone of the fluidized bed. This is where devolatilization reactions occur intensively, see Figure 5 (b) - an example of reaction rate distribution at steady state. It is clear that the biomass particles are heated rapidly in the dense zone and begin to reach the pyrolysis temperature at around $500 \mathrm{~K}$ a short distance away from the injection point. In the free board of the fluidized bed, the density gradient of the biomass particle is much lower as most of the devolatilization reactions are finished in the dense zone. In addition, the apparent density profile is different depending on the shrinkage pattern. In the case of no shrinkage, the apparent density drops from $400 \mathrm{~kg} / \mathrm{m}^{3}$ at the inlet of the reactor to $95 \mathrm{~kg} / \mathrm{m}^{3}$ at the outlet. In the case of shrinkage patterns 1 and 2, the values at the outlet are $160 \mathrm{~kg} / \mathrm{m}^{3}$ and $245 \mathrm{~kg} / \mathrm{m}^{3}$, respectively. Obviously, the highest level of apparent density decrease appears in the case of no shrinkage pattern, then shrinkage pattern 1 and finally shrinkage pattern 2 . This is because biomass decomposition in the case of no shrinkage leads wholly to pore formation, while in the other two cases particle size decrease is also the result of the biomass decomposition. The apparent density of the biomass particle could actually increase and exceed the initial value of $400 \mathrm{~kg} / \mathrm{m}^{3}$, if smaller values of $\alpha$ and $\gamma$ were used (for example $\alpha=0.2, \gamma=0.2$ ). This is because smaller values of $\alpha$ and $\gamma$ represent a larger degree of solid matrix reconstitution during the char formation process and lesser degree of pore formation respectively, which is equivalent to a dominant reconstitution of the solid matrix during the process of biomass pyrolysis. These results are not shown here.

$$
\bar{\rho}_{\text {apparent }, y}=\frac{\iint_{t x} \varepsilon_{x, y} \rho_{\text {apparent }, y} d V d t}{\left(t_{2}-t_{1}\right) \int \varepsilon_{x, y} d V}
$$



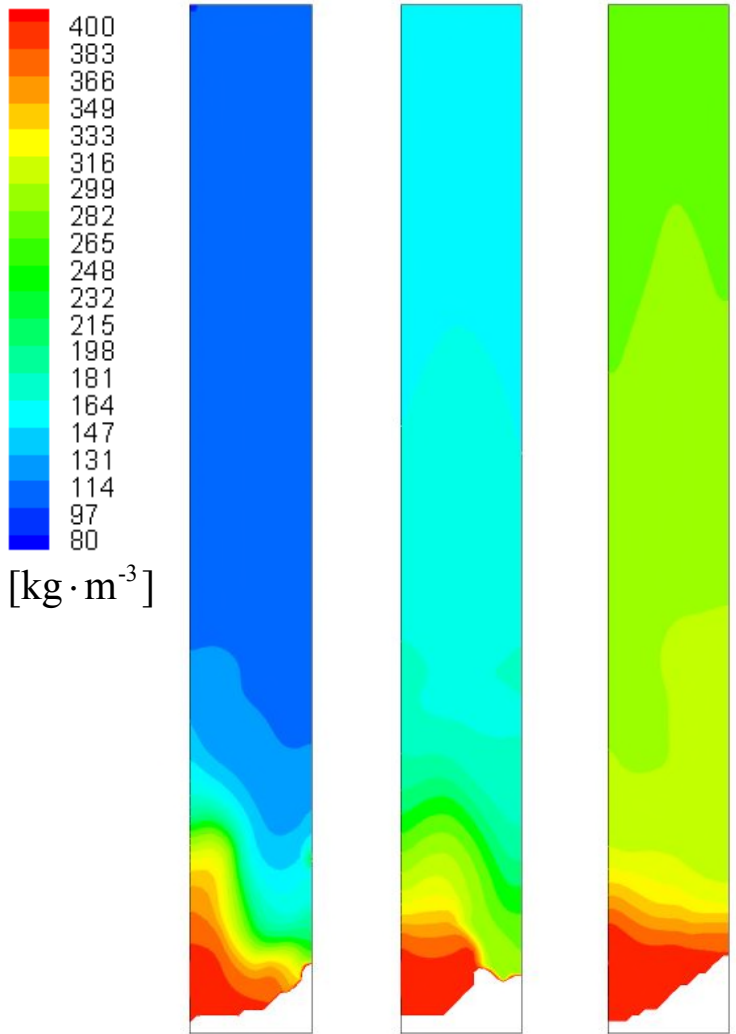

(a)

(b)

(c)

Fig. 6 Apparent density distribution of the biomass particles inside the fluidized bed at 24s: (a) no shrinkage pattern; (b) shrinkage pattern 1; (c) shrinkage pattern 2

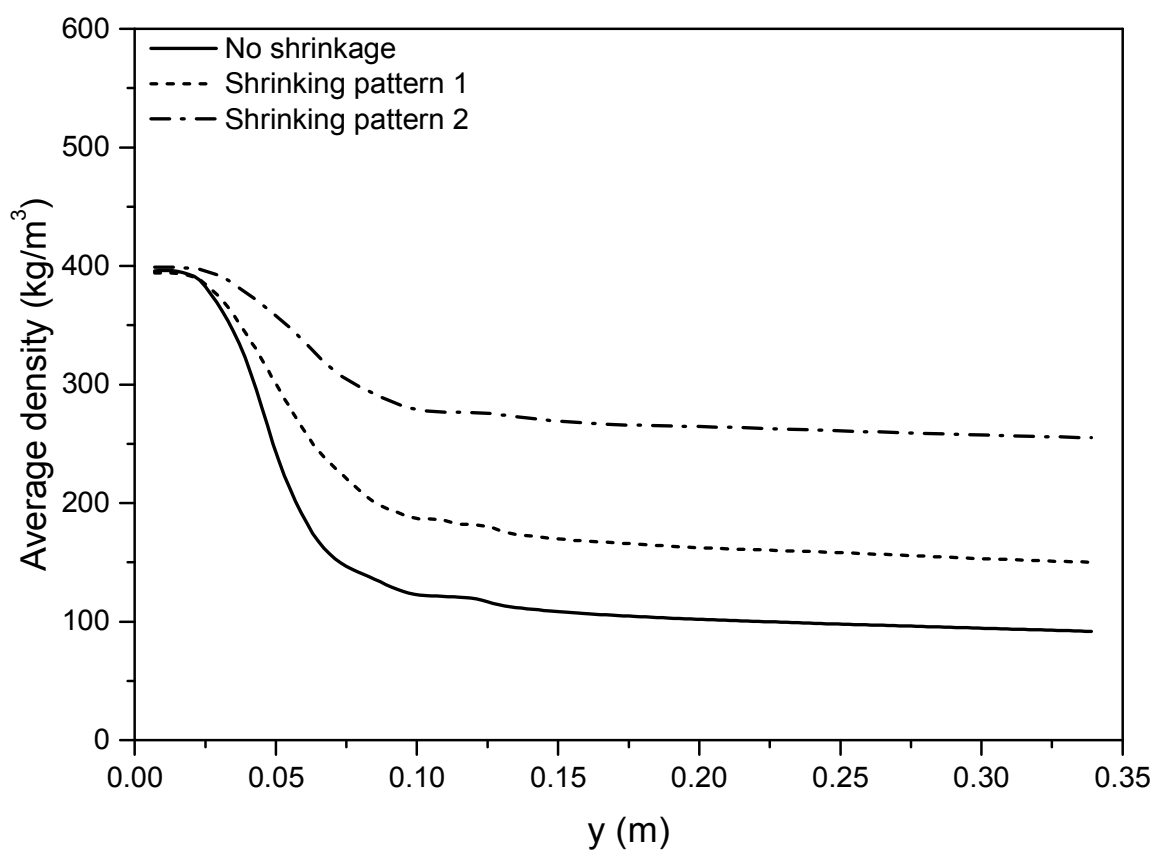

Fig. 7 Spatial-temporal averaged density of the biomass particles in different shrinkage patterns along the $y$ axis

481 Figure 7 gives a statistical average result of the biomass density variation in the fluidized bed reactor 
the volume-fraction-weighted mean density at each cross section of the fluidized bed and averaging it over a period of time after steady state was finally achieved (see equation 12). Oscillations of the stochastic instantaneous results were smoothed. These results give the apparent density evolution of the biomass particles quantitatively in the fluidized bed and in good agreement with the phenomena exhibited in Figure 6.

Particle size evolution for different shrinking patterns is illustrated in Figure $8.0^{\text {th }}-5^{\text {th }}$ moment conservation equations of the biomass phase were solved together with all other transport equations synchronously; and the Sauter mean diameter of the biomass phase was derived from $m_{3} / m_{2}$ for every control volume in the computational domain. As expected, particle size remained constant and equal to the initial value at any point within the domain in the case of no shrinking pattern. In the case of shrinking patterns 1 and 2, the particle size decreased related to the progress of the biomass decomposition. Similarly to the apparent density, the highest gradient of particle shrinkage was observed near the inlet area where the devolatilization and heterogeneous reactions mainly occur. It was also observed that the temperature profile of the biomass phase was insensitive to different shrinkage patterns in the current study. Figure 9 gives the spatial-temporal averaged temperatures of the biomass phase along the $y$ axis of the fluidized bed. It can be seen that curves representing different shrinkage patterns overlap with each other in most parts of the reactor. Figure 10 gives the spatial-temporal-averaged value of the biomass particle diameter along the $y$ axis of the fluidized bed. Simulation results show that the average diameter of the biomass particle drops to $290 \mu \mathrm{m}$ and $250 \mu \mathrm{m}$ near the outlet of the fluidized bed for shrinking patterns 1 and 2, respectively. The pattern in which

503 a biomass particle shrinks not only depends on the physical properties of the particle itself, such as 504 size, shape, composition, structure, etc., but also on the surrounding heat and mass transfer environment. Like some researchers in their work $[28,34]$, the shrinkage factors investigated in the current study were given arbitrarily. To our knowledge, no current work gives an exact correlation between the shrinkage factor and a specific type of biomass particle. Therefore, this work focuses 

predicting an exact result for a real process.

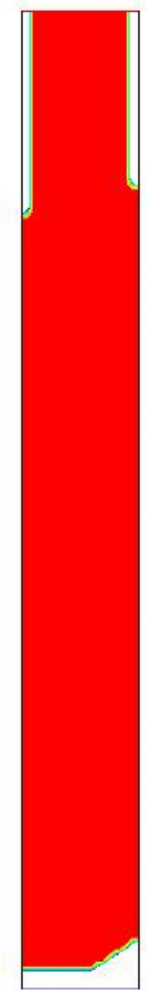

(a)

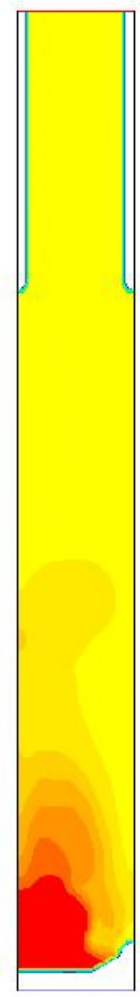

(b)

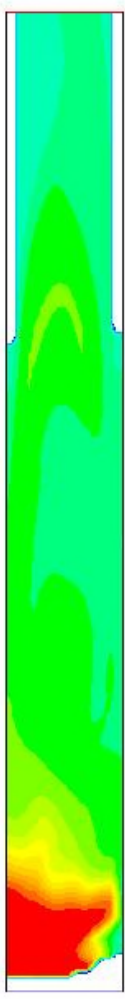

(c)

Fig. 8 Size distribution of the biomass particles inside the fluidized bed at 24s: (a) no shrinkage pattern; (b) shrinkage pattern 1; (c) shrinkage pattern 2

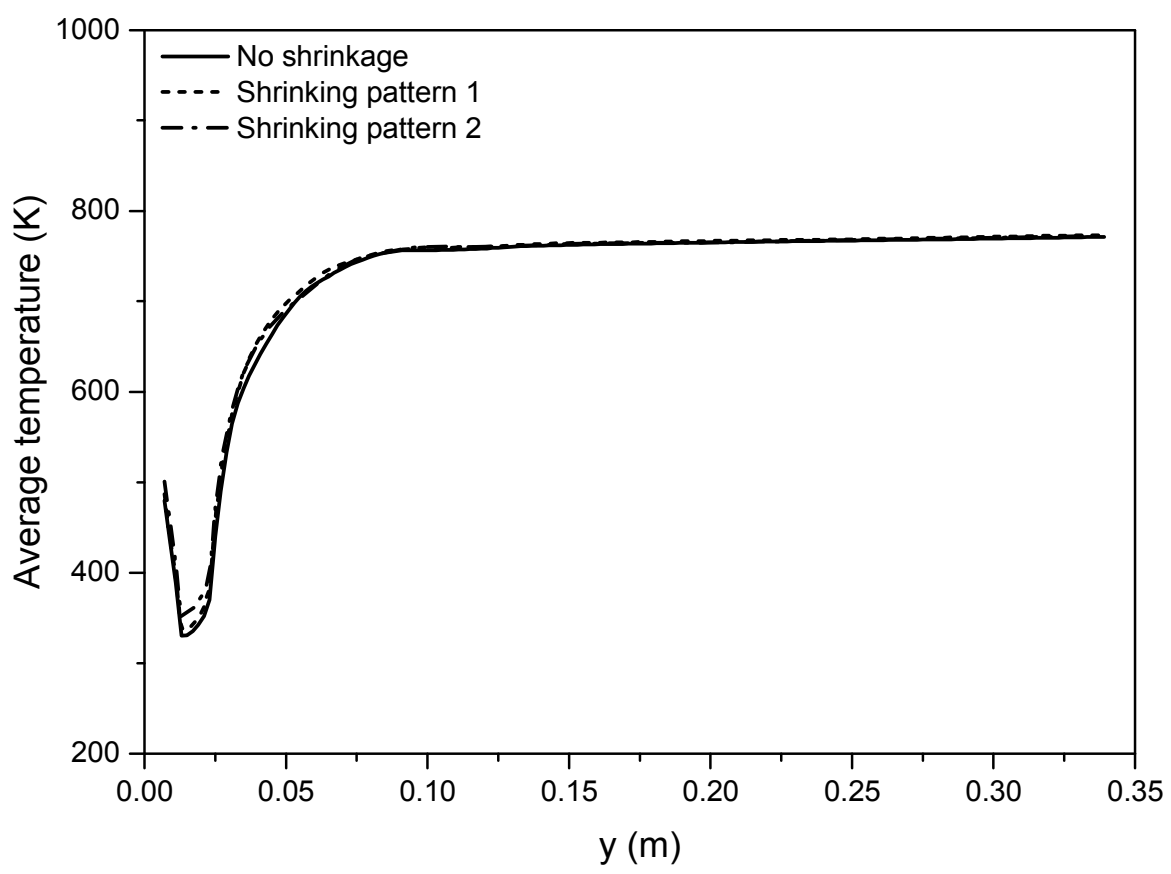

Fig. 9 Spatial-temporal averaged temperature of the biomass particles in different shrinkage patterns along the $y$ axis 




Fig. 10 Spatial-time averaged diameter of the biomass particles in different shrinkage patterns along the $y$ axis

\subsection{Particle tracking in an Eulerian CFD framework}

Knowledge of particle motion is of great importance for the design of fluidized bed reactors, since it can help to optimize the reactor configuration and choose reasonable operating conditions. In general, two methods are used to simulate particle laden flows in a fluidized bed reactor, the Eulerian-granular model and the Discrete Element Method DEM-CFD model. The latter is more suitable for particle trajectory calculation as the complex fluid-particle and particle-particle interactions are investigated by tracking a great number of 'real' particles individually; however, this method is extremely computational expensive. In contrast, the computing cost of the Eulerian-granular method is much lower because the particles are considered as a continuum, and particle-particle and particle-fluid interactions are accounted for by means of solid viscosity closured by the granular kinetic theory and drag force models, respectively. Particle tracking is also possible under the Eulerian-granular framework, which is referred as the 'fictitious tracer particle' concept proposed by Liu and Chen [64]. The concept of massless particle, routinely used by many researchers [65-67], is based on the calculation of the trajectory of a moving Lagrangian element with the knowledge of an Eulerian flow 
field. The tracer velocity can be either assigned by the local velocity of the nearest cell or extracted with high-order numerical schemes [68-70]. Each fictitious tracer particle can be laid in an interested computational cell at a specific physical simulation time and fully follow the movement of the solid phase. The tracer particle is massless and the velocity is assigned by the solid phase velocity of the cell where the tracer particle is located. Thus, the displacement of the tracer particle can be calculated after a time interval, and a new position of the particle is then obtained. When this procedure is repeated within each time step during the simulation, the trajectory of each tracer particle is obtained. Although this is not a rigorous particle tracking technology based on 'real' particles, some important particle motion information can be obtained. Forces exerted on the tracer particle, such as fiction and collision, are implied in the solid phase motion accounted for by the kinetic theory.

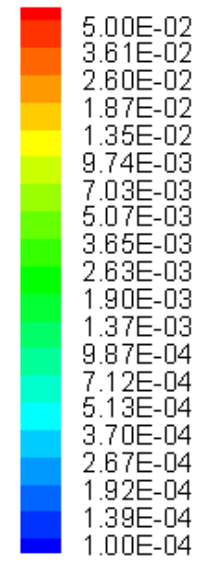

Fig. 11 (a) The biomass volume fraction and (b) comparison of the particle trajectories in the fluidized bed at $24 \mathrm{~s}$ (no shrinkage pattern)

551 fluidized bed. These variables are closely related to parameters such as biomass conversion, char 

of the superficial velocity of the fluidized gas and the gas phase effective volume in the fluidized bed. However, solid residence time cannot be estimated in this way, since particles shrink and flow back far more frequently than in the gaseous phase. In order to estimate the solid residence time of the fluidized bed, 'fictitious tracer particle' concept was applied in this work. Massless tracer particles were released one by one at different positions near the biomass inlet for every 50 time steps and tracked in accordance with the transient update of the flow field. The velocity of an individual tracer particle is assigned by the local velocity of the biomass phase at the control volume where the particle is currently located. If the tracer particle moves and touches the walls, a full elastic reflection happens so that a continuous particle trajectory can be obtained until the particle leaves the domain from the top outlet. Most of the particles leave the domain after a certain period of tracking. However, a few may be trapped permanently because zero-velocity regions exist at the two sides close to the outlet of the fluidized bed. In addition to residence time, other particle information can be recorded dynamically with this tracking technology. Any of the parameters of interest can be stored in a data matrix when implementing the tracking process, similarly to that of storing the position information, such as particle temperature, local sand volume fraction, etc. Some statistically averaged characteristics of the biomass phase behaviour can be obtained by tracking a large number of individual particles in a fluidized bed. Figure 11 gives a comparison of biomass volume fractions alongside a number of particle trajectories. It can be observed that regions with dense particle trajectories correspond accordingly with high volume fractions derived from the VOF equation.

572 Particles entering the domain with a low initial velocity from the inlet are unlikely to spread 573 immediately across a wide range within the fluidized bed medium. Instead, most of them travel along 574 the side wall to the top of the dense zone until reaching the splashing zone where more intensive 575 mixing happens due to bubble breakage; hence particles spread faster throughout the whole splashing zone. 




(a)

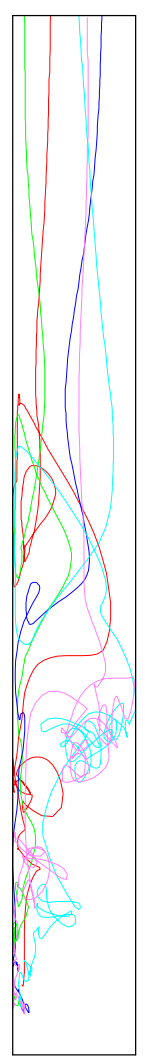

(b)

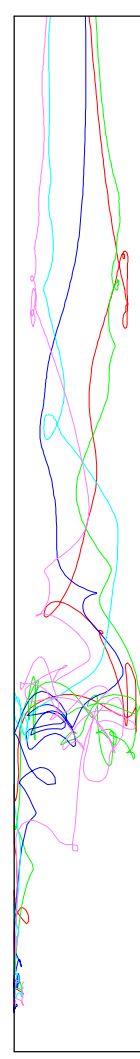

(c)

Fig. 12 Typical particle trajectories of the biomass particle in different shrinking pattern: (a) no shrinkage pattern; (b) shrinkage pattern 1; (c) shrinkage pattern 2

Differences in the biomass particle motion inside the fluidized bed due to the implementation of a different particle shrinkage pattern can be observed by means of the trajectories of the particles.

Figure 12 shows typical particle trajectories for different shrinkage patterns. It can be seen that, when no shrinkage pattern is considered, most of the particles are likely to escape the splashing zone into the free board without significant flow back (such as the red, blue and green lines in Figure 12(a)). Nevertheless, some of the particles cannot directly escape the splashing zone and stay for some time before entering the free board area (cyan and purple lines in Figure 12(a)). In the case of shrinkage pattern 1, the flow back of the particles in the splashing zone and the area immediately after this zone is more frequent. This may be related to the higher particle density which seems then to have a higher influence on the particle motion than that of the particle size. In the case of particle shrinkage pattern 2 , few particles escape the splashing zone directly. Most are trapped in the splashing zone for different periods of time before entering the free board. Particles undergoing shrinkage pattern 2 flow back 
frequently, even once they are in the free board area and near to the outlet of the reactor (see Figure

594

595

596

597

598

599

600

601

602

603

604

605

606

607

608

609

610

611

612

613

614

615

616 12(c)).

Figure 13 shows the spatial-temporal-average y-velocity of the biomass particles inside the fluidized bed. In the case of no shrinkage pattern and shrinkage pattern 1, the $y$-velocity of the biomass phase is positive, on average, across the splashing zone, which means that back flow is not dominant. In the free board, the velocity observed when no shrinkage is implemented is slightly higher than that of shrinkage pattern 1, leading to a slightly shorter residence time. In contrast, in the case of shrinkage pattern 2, the average $y$-velocity is negative across the splashing zone which shows significant back flow and longer residence time of the particles in this area.

Figure 14 (a) gives a comparison of the biomass particle residence time in the dense zone. This was obtained by recording the time when the volume fraction of the sand phase becomes zero during the particle tracking process. Over 200 particles were tracked for each shrinkage pattern to obtain statistically average values for the residence time. Bar diagrams were drawn by counting the number of particles for specific time intervals. As can be observed, in the case of the no shrinkage pattern, a major peak appears at around $1.8 \mathrm{~s}$ on the curve of the number density distribution. The majority of particles $(71 \%)$ in this case leave the dense zone in up to $2.8 \mathrm{~s}$. This peak occurs slightly later in the case of shrinkage pattern 1 , at about 2.4s. In addition, a second obvious peak arises around 3.6s. This situation can be attributed to the non-negligible number of particles trapped when passing through the splashing zone. Therefore, the residence time in this case increases up to $3.6 \mathrm{~s}$ for about $74 \%$ of the total particles tracked. In the case of shrinkage pattern 2, this proves to be much more common as most of the biomass particles are trapped in the splashing zone and eventually escape only by chance. Hence, more than one peak appears, and the whole probability density profile distributes more evenly than the other two shrinkage patterns. As a result, particle residence time increases more in this case, and $73 \%$ of the tracer particles leave the dense zone in $4.7 \mathrm{~s}$. 
617 The total residence time of the biomass particles in the whole domain can be similarly estimated by

618 recording the time when a particle approaches the outlet of the reactor. Figure 14 (b) gives the 619 probability density distribution of the total particle residence time for this domain. For the no 620 shrinkage pattern, a major peak appears around 2.9s, followed by two other small peaks around 4.8s 621 and 5.6s, respectively. For shrinkage pattern 1, two peaks of similar size appear at about 3.2s and 4.4s, 622 respectively. For shrinkage pattern 2 , five obvious peaks appear at $4 s, 4.8 s, 5.6 s, 9.2 s$ and $10.8 s$, 623 representing different particle escape modes from the splashing zone.

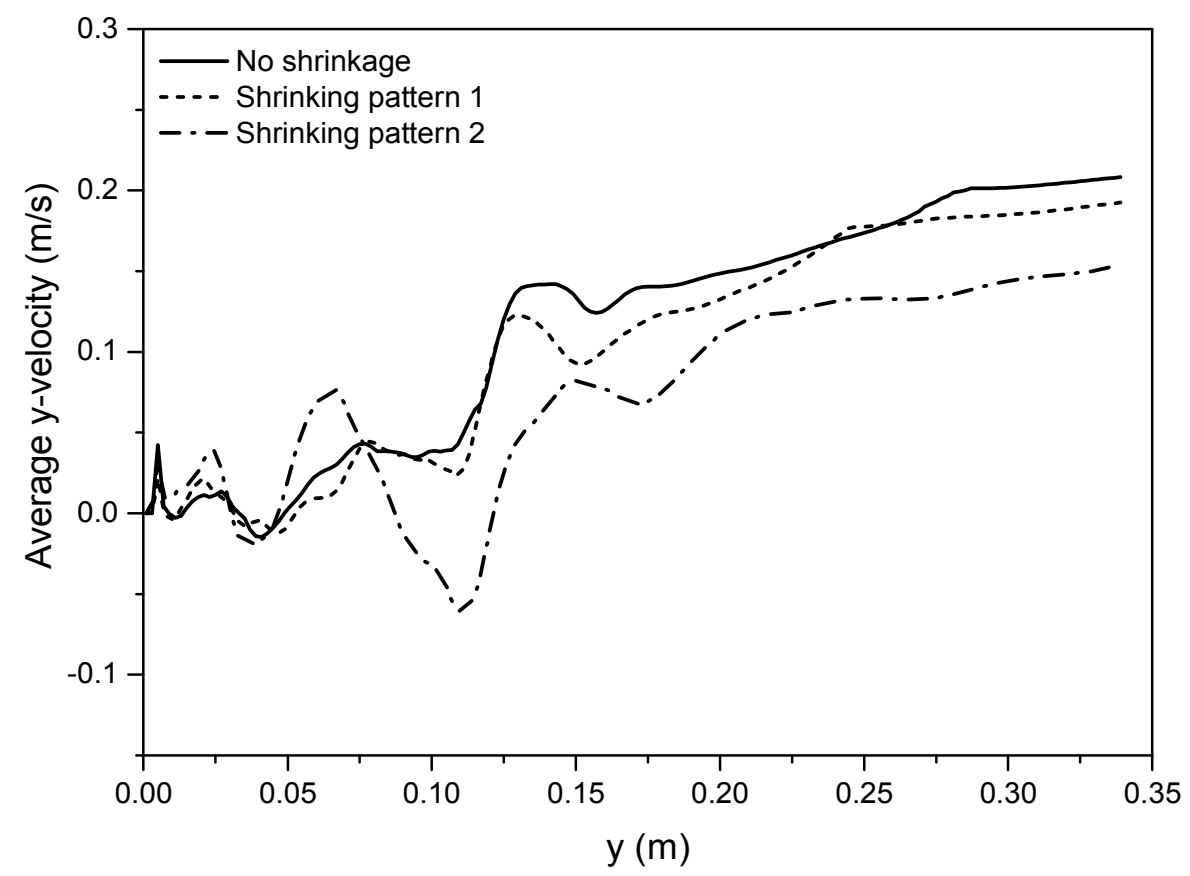




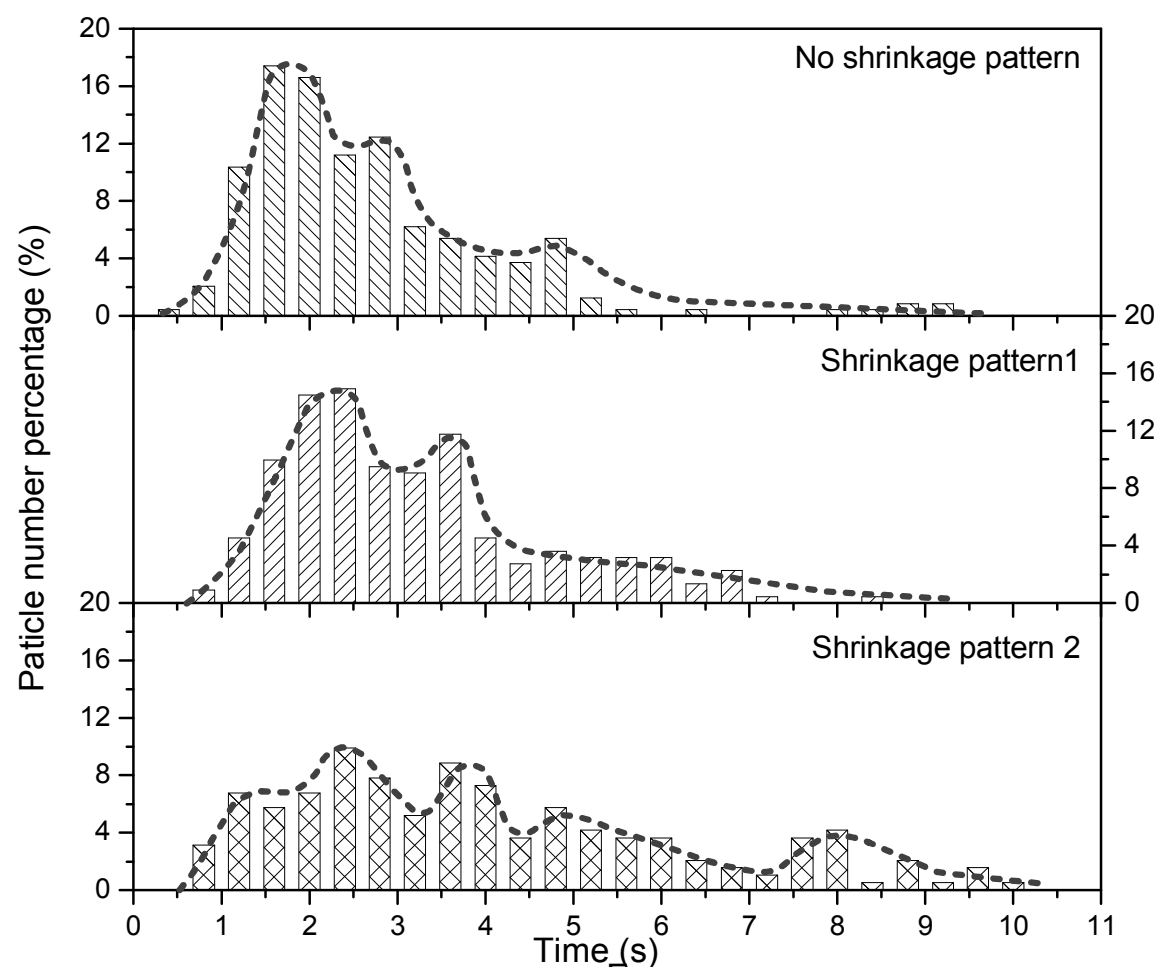

(a)

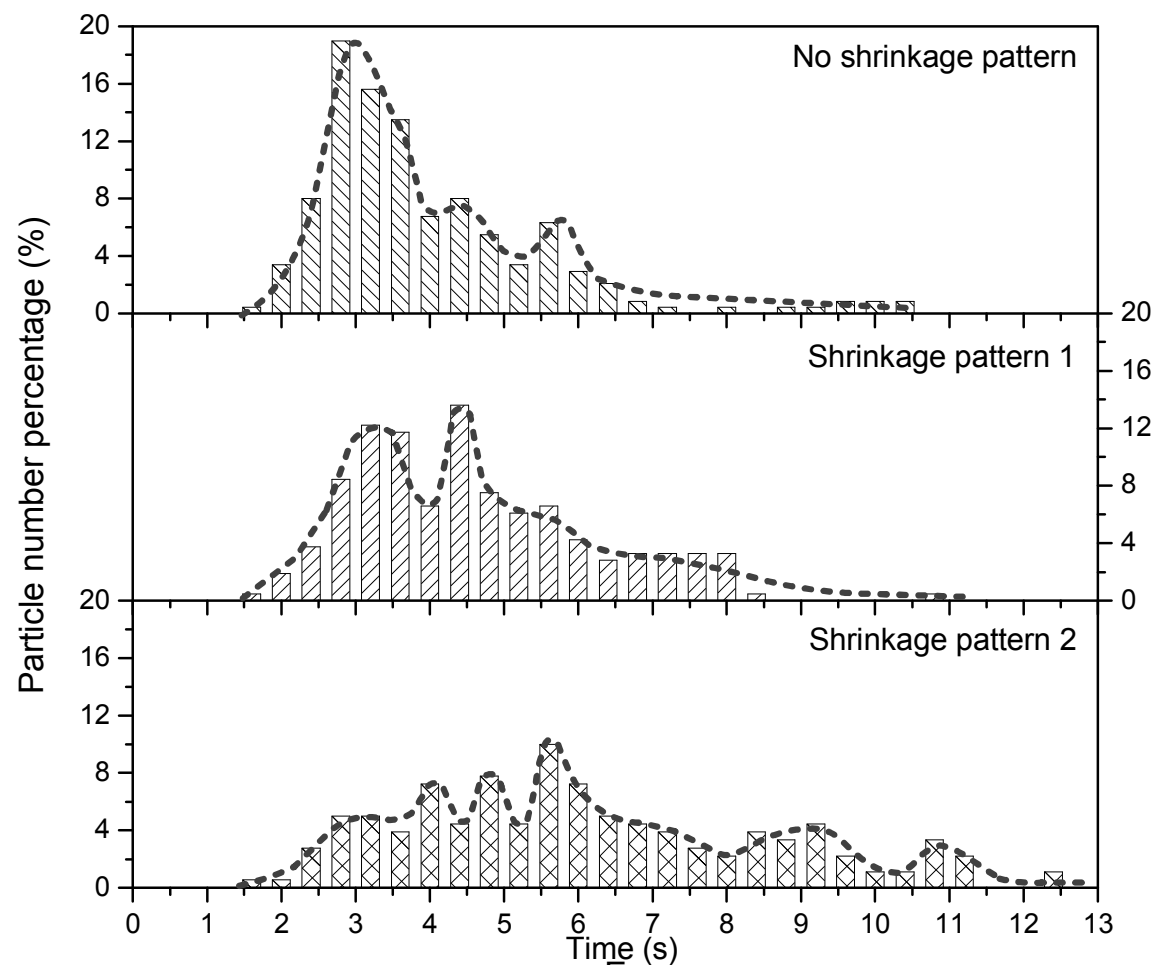

Fig. 14 Residence time of the biomass particles in (a) the dense zone and (b) the whole 
633 It should be pointed out that these characteristic time values are rough estimations as the total number of the tracer particles are limited. Moreover, in the case of shrinkage pattern 2, about $10 \%$ of the tracer particles were still trapped in the splashing zone when tracking terminated, due to the preset maximum tracking time limit being reached. Based on these particle tracking data, the numberweighted-average residence time was calculated and is shown in Table 9. This provides an outline of the effects of shrinkage patterns on particle residence times.

Table 9 Particle average residence time

\begin{tabular}{lcc}
\hline Shrinkage pattern & Residence time (dense zone(s)) & Residence time (domain(s)) \\
\hline No shrinkage & 2.69 & 3.96 \\
Pattern 1 & 3.17 & 4.60 \\
Pattern 2 & 4.04 & 5.73 \\
\hline
\end{tabular}

640

\subsection{Product yields of different shrinkage patterns}

642 Results for product yields were compared to results obtained from experiments carried out in reactors 643 with similar geometry, feedstock composition (cellulose: 32-52\%; hemicellulose: 23-33\%; lignin: 13$64427 \%)$ and operating conditions to those used in this work $[22,71]$. The simulation results for the different particle shrinkage patterns and experimental results are compared in Table 10. The product yields from the model are calculated as an average of each of the species flow rate at the outlet of the fluidized bed. In general, simulation results are in good agreement with the experimental results, especially those obtained by Patel [71]. It can be observed that the bio-oil yield seems insensitive to particle shrinkage patterns, which is approximately 63\%. However, significant changes in noncondensable gas and char yields are observed depending on the shrinkage pattern applied (i.e. 1 or 2). Indeed, the application of shrinkage pattern 2 gave rise to higher biomass conversion and char yield than that obtained with pattern 1 and with no shrinkage pattern. This might be attributed to the longer residence time of the biomass particle, which has been discussed in section 4.4. From the three main constituents of the biomass, hemicellulose has the most reaction activity, then cellulose; lignin has 

of lignin, which is known to lead to higher char yields [72].

Table 10 Product yields (experiments from literature and simulation result at different shrinkage patterns)

\begin{tabular}{|c|c|c|c|c|c|c|c|}
\hline Method & Feed stock & $\begin{array}{l}\text { Bio-oil yield } \\
\text { (wt. \%) }\end{array}$ & $\begin{array}{l}\text { Non-condensable } \\
\text { gas yield (wt. \%) }\end{array}$ & $\begin{array}{l}\text { Char yield } \\
\text { (wt. \%) }\end{array}$ & $\begin{array}{l}\text { Residue } \\
\text { (wt. \%) }\end{array}$ & $\begin{array}{l}\text { Temperature } \\
\text { (K) }\end{array}$ & $\begin{array}{l}\text { Particle size } \\
(\mathrm{mm})\end{array}$ \\
\hline Experiment $^{a}$ & $\begin{array}{l}\text { Beech } \\
\text { wood }\end{array}$ & 63.87 & 11.69 & 14.83 & & 779-798 & $0.25-1.0$ \\
\hline Experiment ${ }^{b}$ & $\begin{array}{l}\text { Bagasse } \\
\text { pallet }\end{array}$ & 60.45 & 14.01 & 17.32 & & 779-798 & $0.25-1.0$ \\
\hline $\begin{array}{l}\text { Experiment }{ }^{\mathrm{c}} \\
\text { Simulation }\end{array}$ & Red oak & $71.7 \pm 1.4$ & $20.5 \pm 1.3$ & $13.0 \pm 1.5$ & - & 773 & $0.25-0.4$ \\
\hline no shrinkage & - & 62.83 & 11.18 & 14.93 & 10.76 & $771.45^{d}$ & $0.325^{e}$ \\
\hline $\begin{array}{l}\text { Simulation } \\
\text { shrinkage } 1\end{array}$ & - & 63.40 & 10.49 & 15.50 & 10.47 & $773.56^{d}$ & $0.325^{e}$ \\
\hline $\begin{array}{l}\text { Simulation } \\
\text { shrinkage } 2\end{array}$ & - & 63.38 & 12.18 & 17.83 & 6.51 & $772.46^{d}$ & $0.325^{e}$ \\
\hline
\end{tabular}

Note: a, b - experimental data from Patel, 2013; c - experimental data from Xue et al., 2012; d - outlet temperature

e - Sauter mean diameter

(a)

(b)

(c) 
Figure 15 shows the distribution of the mass fraction of char along the fluidized bed at $24 \mathrm{~s}$ for the three shrinkage patterns. It can be observed clearly that the mass fraction of char near the outlet increases with the degree of particle shrinkage. This is because the more the particles shrink, the longer their residence time in the reactor, which favours the conversion of lignin towards char. The results shown in Figure 15 are in good agreement with the evidence of the relationship between particle residence time and shrinkage patterns provided in section 4.4.

\section{5. Conclusion}

In this paper, biomass particle shrinkage in a lab-scale fluidized bed was successfully simulated with a comprehensive CFD model. The state-of-the-art QMOM method for solving the particle PBE was employed, accounting for particle size evolution in the fluidized bed. A 3-parameter shrinkage model was used to calculate the particle shrinking rate as well as the apparent density of the biomass particle. Three different sets of shrinkage factors related to different particle shrinkage patterns were investigated thoroughly to determine how size and density variation affect the main performance parameters of the fluidized bed, such as product yields, char distribution, particle residence time, etc. The degree of shrinkage increases in the sequence of no shrinkage pattern, shrinkage pattern 1 and shrinkage pattern 2 , in which the shrinkage factor takes the value of $\alpha=1, \beta=1, \gamma=1 ; \alpha=1, \beta=0, \gamma=1$; and $\alpha=0.5, \beta=0, \gamma=0.5$, respectively.

It was shown that for all three cases investigated in this study, the particle apparent density decreases due to continuous mass loss and pore formation. Particle apparent density at the outlet of the fluidized bed drops to $95 \mathrm{~kg} / \mathrm{m}^{3}, 160 \mathrm{~kg} / \mathrm{m}^{3}$ and $245 \mathrm{~kg} / \mathrm{m}^{3}$ correspondingly from an initial value of $400 \mathrm{~kg} / \mathrm{m}^{3}$. In the no shrinkage case, mass loss of the biomass particle was totally accounted for by pore formation so that a constant size could be maintained, while, in the other two cases, this was only partly accounted for by pore formation, and depends in part on the particle shrinkage. Hence, the particle diameter at the outlet comes to $290 \mu \mathrm{m}$ and $250 \mu \mathrm{m}$ respectively, due to the different percentage of distribution of the aforementioned particle phenomena. 
An innovative particle tracking technology based on the Eulerian CFD framework is demonstrated in this study to quantitatively estimate the particle residence time. A number of individual particles were consistently tracked from a dynamic update of the flow field. The biomass particle residence time in the dense zone and the whole domain were evaluated statistically with the tracking results. On average these were $2.69 \mathrm{~s}, 3.17 \mathrm{~s}, 4.04 \mathrm{~s}$ for the dense zone, and 3.96s, 4.6s, $5.73 \mathrm{~s}$ for the whole domain. explanation for the effect of some of the characteristics of the fluidized bed that arise from variations in the particle properties.

Different particle shrinkage patterns also have an effect on the devolatilization process of the biomass reactants, especially the percentage of conversion and char distribution. Bio-oil yield seems insensitive to particle shrinkage patterns, at approximately $63 \%$. In contrast, char yield increases slightly with the degree of particle shrinking, from $14.93 \%$ for no shrinkage pattern to $17.83 \%$ for shrinking pattern 2 . This is due to the increasing residence time of biomass particles.

\section{Acknowledgement} international staff exchange in the UK. 
$713 \bar{d}$ - particle number-mean diameter $(\mathrm{m})$

$714 D_{i, j}$ - mass diffusion coefficient of the $j^{\text {th }}$ component in the $i^{\text {th }}$ phase $\left(\mathrm{kg} \cdot \mathrm{m}^{-1} \cdot \mathrm{s}^{-1}\right)$

$715 E$ - activated energy in the Arrhenius equation $\left(\mathrm{kJ} \cdot \mathrm{mol}^{-1}\right)$

$716 e_{s s}-$ the restitution coefficient

$717 g_{0, s s}-$ the radial distribution function

$718 h$ - phase enthalpy $\left(J \cdot \mathrm{kg}^{-1}\right)$

$719 h_{i, k}-$ interphase heat transfer coefficient $\left(\mathrm{W} \cdot \mathrm{K}^{-1} \cdot \mathrm{m}^{-3}\right)$

$720 \Delta h$ - enthalpy change $\left(\mathrm{kJ} \cdot \mathrm{kg}^{-1}\right)$

$721 I_{2 D}-$ the second invariant of the deviatoric stress tensor

$722 \overline{\bar{I}}$ - unit tensor

$723 g-$ acceleration of gravity $\left(\mathrm{m} \cdot \mathrm{s}^{-2}\right)$

$724 \quad k$-reaction rate $\left(\mathrm{kg} \cdot \mathrm{m}^{-3} \cdot \mathrm{s}^{-1}\right)$

$725 K_{i, j}$ - phase exchange coefficient of momentum $\left(\mathrm{kg} \cdot \mathrm{m}^{-3} \cdot \mathrm{s}^{-1}\right)$

$726 L$ - particle size $(\mathrm{m})$

$727 \quad M-$ mass $(\mathrm{kg})$

$728 m_{i}-$ the $i^{\text {th }}$ moment of the biomass phase

$729 m_{i, j}$ - mass transfer rate from phase $i$ to phase $j\left(\mathrm{~kg} \cdot \mathrm{m}^{-3} \cdot \mathrm{s}^{-1}\right)$

$730 m_{k^{l}, i^{j}}-$ mass transfer rate per unit volume of the $i^{\text {th }}$ component of the $k^{\text {th }}$ phase to the $j^{\text {th }}$ component

731 of the $i^{\text {th }}$ phase $\left(\mathrm{kg} \cdot \mathrm{m}^{-3} \cdot \mathrm{s}^{-1}\right)$

$732 N$ - total number of the biomass particle per unit volume

$733 N u-$ Nusselt number

$734 p$-pressure $(\mathrm{Pa})$

$735 \quad P r-$ Prandtl number

$736 Q_{i, j}$ - interphase heat transfer rate between phase $i$ and phase $j\left(\mathrm{~J} \cdot \mathrm{m}^{-3} \cdot \mathrm{s}^{-1}\right)$

$737 R$-volume shrinkage rate $\left(\mathrm{m}^{3} \cdot \mathrm{m}^{-3} \cdot \mathrm{s}^{-1}\right)$ 
$\mathbf{R}_{i, j}$ - drag force between phase $i$ and phase $j\left(\mathrm{~N} \cdot \mathrm{m}^{-3}\right)$

739

$\mathrm{R}_{i, j}$ - net producing rate of the $j^{\text {th }}$ component in the $i^{\text {th }}$ phase $\left(\mathrm{kg} \cdot \mathrm{m}^{-3} \cdot \mathrm{s}^{-1}\right)$

740

Re - Reynolds number

741

$S$ - source term of the conservation equations

742

$t-$ time $(\mathrm{s})$

743

$T$ - temperature (K)

$744 \mathbf{u}$ - velocity vector $\left(m \cdot s^{-1}\right)$

$745 \quad V$-volume $\left(\mathrm{m}^{3}\right)$

$746 x_{i, j}$ - mass fraction of $j^{\text {th }}$ component of the $i^{\text {th }}$ phase

$747 \quad Y$-stoichiometric coefficient of char

\section{$748 \quad$ Greek letters}

749

$\alpha$ - shrinkage factor

750

B - shrinkage factor

751

$\gamma$ - shrinkage factor

752

$\varepsilon$-volume fraction of a specific phase

753

$\varphi$ - angle of internal friction

754

$\lambda_{s}-$ bulk viscosity of a solid phase (Pa.s)

755

$\lambda_{i}$ - phase thermal conductivity coefficient $\left(\mathrm{W} \cdot \mathrm{m}^{-1} \cdot \mathrm{K}^{-1}\right)$

756

$\lambda$-species thermal conductivity coefficient $\left(\mathrm{W} \cdot \mathrm{m}^{-1} \cdot \mathrm{K}^{-1}\right)$

$757 \mu-\operatorname{viscosity~(Pa\cdot s)}$

$758 \mu_{s, c o l}-$ collision viscosity of a solid phase (Pa.s)

759

$\mu_{s, k i n}$ - kinetic viscosity of a solid phase (Pa.s)

760

$\mu_{s, f r}-$ frictional viscosity of a solid phase (Pa.s)

761

$\rho$ - density $\left(\mathrm{kg} \cdot \mathrm{m}^{-3}\right)$

762

$\rho_{\text {apparent }}-$ apparent density $\left(\mathrm{kg} \cdot \mathrm{m}^{-3}\right)$

$763 \overline{\bar{\tau}}-$ Stress tensor of a specific phase momentum equation 
$765 \quad \eta$-reaction progress factor

\section{Subscripts}

$767 b$-biomass phase

$768 c$-cellulose

$769 h$-hemicellulose

$770 \quad 1-$ lignin

$771 \quad C-$ char

$772 g$-gas

$773 i$-common index of component or phase

$774 j$-common index of component or phase

$775 s-$ solid, sand

$776 \quad W$ - biomass solid 
[1] Sawin, J.L., Sverrisson, F., Seyboth, K., Adib, R., Murdock, H.E., Lins, C., Brown, A., Di Domenico, S.E.,

Kielmanowicz, D., Williamson, L.E. \& Jawahar, R., Renewables 2016 Global Status Report. Key findings.

A Record Breaking Year for Renewable Energy: New Installations, Policy Targets, Investment and Jobs.

781

782

783

784

785

786

787

788

789

790

791

792

793

794

795

796

797

798

799

800

801
Mainstreaming renewables: guidance for policy makers.

[2] Huber, G.W. \& Corma, A., 2007. Synergies between bio- and oil refineries for the production of fuels from biomass, Angewandte Chemie - International Edition 46(38), 7184-7201.

[3] Zinoviev, S., Muller-Langer, F., Das, P., Bertero, N., Fornasiero, P., Kaltschmitt, M., Centi, G. \& Miertus, S., 2010. Next-generation biofuels: Survey of emerging technologies and sustainability issues, ChemSusChem 3(10), 1106-1133.

[4] Bridgwater, A.V., 2003. Renewable fuels and chemicals by thermal processing of biomass, Chemical Engineering Journal 91(2), 87-102.

[5] Bridgwater, A.V., 1995. The technical and economic feasibility of biomass gasification for power generation, Fuel, 74(5), 631-653.

[6] Laird, D.A., Brown, R.C., Amonette, J.E. \& Lehmann, J., 2009. Review of the pyrolysis platform for coproducing bio-oil and biochar, Biofuels, Bioproducts and Biorefining 3(5), 547-562.

[7] Wright, M.M. \& Brown, R.C., 2007. Comparative economics of biorefineries based on the biochemical and thermochemical platforms, Biofuels, Bioproducts and Biorefining 1(1), 49-56.

[8] Czernik, S. \& Bridgwater, A.V., 2004. Overview of applications of biomass fast pyrolysis oil, Energy \& Fuels 18(2), 590-598.

[9] Mohan, D., Pittman, C.U. \& Steele, P.H., 2006. Pyrolysis of Wood/Biomass for Bio-oil: A Critical Review, Energy \& Fuels 20(3), 848-889.

[10] Scott, D.S., Majerski, P., Piskorz, J. \& Radlein, D., 1999. A second look at fast pyrolysis of biomassthe RTI process, Journal of Analytical and Applied Pyrolysis 51(1), 23-37.

[11] Pepiot, P., Dibble, C. J. \& Foust, T.D., 2010. Computational fluid dynamics modeling of biomass 
gasification and pyrolysis, Computational modeling in lignocellulosic biofuel production. Golden:

803

804

805

806

807

808

809

810

811

812

813

814

815

816

817

818

819

820

821

822

823

824

825

826

827

American Chemical Society, 273-298.

[12] Xue, Q. \& Fox, R.O., 2015. Computational Modeling of Biomass Thermochemical Conversion in Fluidized Beds: Particle Density Variation and Size Distribution, Industrial \& Engineering Chemistry Research 54(16), 4084-4094.

[13] Fan, R. \& Fox, R.O., 2008. Segregation in polydisperse fluidized beds: Validation of a multi-fluid model, Chemical Engineering Science 63(1), 272-285.

[14] Kuipers, J. A.M. \& van Swaaij, W.P.M., 1998. Computational fluid dynamics applied to chemical reaction engineering, Advances in Chemical Engineering 24, 227-328.

[15] van der Hoef, M.A., van Sint Annaland, M., Deen, N.G. \& Kuipers, J.A.M., 2008. Numerical Simulation of Dense Gas-Solid Fluidized Beds: A Multiscale Modeling Strategy, Annual Review of Fluid Mechanics 40(1), 47-70.

[16] Authier, O., Ferrer, M., Mauviel, G., Khalfi, A.E. \& Lédé, J., 2009. Wood fast pyrolysis: Comparison of Lagrangian and Eulerian modeling approaches with experimental measurements, Industrial and Engineering Chemistry Research 48(10), 4796-4809.

[17] Lathouwers, D. \& Bellan, J., 2001. Yield optimization and scaling of fluidized beds for tar production from biomass, Energy and Fuels 15(5), 1247-1262.

[18] Lathouwers, D. \& Bellan, J., 2001. Modeling of dense gas-solid reactive mixtures applied to biomass pyrolysis in a fluidized bed, International Journal of Multiphase Flow 27(12), 2155-2187.

[19] Gerhauser, H., Generalis, S.C., Hague, R.A. \& Bridgwater, A.V., 2001. CFD for the Modelling of Entrainment in Fluidised Bed Fast Pyrolysis of Biomass, Progress in Thermochemical Biomass Conversion, 1281-1295.

[20] Gerber, S., Behrendt, F. \& Oevermann, M., 2010. An Eulerian modeling approach of wood gasification in a bubbling fluidized bed reactor using char as bed material, Fuel 89(10), 2903-2917.

[21] Xue, Q., Heindel, T.J. \& Fox, R.O., 2011. A CFD model for biomass fast pyrolysis in fluidized-bed reactors, Chemical Engineering Science 66(11), 2440-2452. 

modeling study of biomass fast pyrolysis in fluidized-bed reactors, Fuel 97, 757-769.

[23] Mellin, P., Zhang, Q., Kantarelis, E. \& Yang, W., 2013. An Euler-Euler approach to modeling Engineering 58(1), 344-353.

[24] Mellin, P., Kantarelis, E. \& Yang, W., 2014. Computational fluid dynamics modeling of biomass fast

[25] Ranzi, E., Cuoci, A., Faravelli, T., Frassoldati, A., Migliavacca, G., Pierucci, S. \& Sommariva, S., 2008.

Chemical kinetics of biomass pyrolysis, Energy and Fuels 22(6), 4292-4300.

[26] Fletcher, D.F., Haynes, B.S., Christo, F.C. \& Joseph, S.D., 2000. A CFD based combustion model of an entrained flow biomass gasifier, Applied Mathematical Modelling 24(3), 165-182.

[27] Papadikis, K., Gu, S. \& Bridgwater, A.V., 2008. CFD modelling of the fast pyrolysis of biomass in

fluidised bed reactors, Part A: Eulerian computation of momentum transport in bubbling fluidised beds, Chemical Engineering Science 63(16), 4218-4227.

[28] Papadikis, K., Gu, S. \& Bridgwater, A.V., 2009. CFD modelling of the fast pyrolysis of biomass in 149(1), 417-427.

[29] Papadikis, K., Gu, S. \& Bridgwater, A.V., 2009. CFD modelling of the fast pyrolysis of biomass in

[30] Papadikis, K., Gu, S. \& Bridgwater, A.V., 2010. Computational modelling of the impact of particle size to the heat transfer coefficient between biomass particles and a fluidised bed, Fuel Processing Technology 91(1), 68-79.

851 [31] Bruchmülle, J., van Wachem, B.G.M., Gu, S., Luo, K.H. \& Brown, R.C., 2012. Modeling the 852 Thermochemical Degradation of Biomass Inside a Fast Pyrolysis Fluidized Bed Reactor, AIChE Journal 58(10), 3030-3042. 
854 [32] Hastaoglu, M.A., Kahraman, R. \& Syed, M.Q., 2000. Pellet breakup due to pressure generated during wood pyrolysis, Industrial \& Engineering Chemistry Research 39(9), 3255-3263.

[33] Miller, R.S. \& Bellan, J., 1998. Numerical Simulation of Vortex Pyrolysis Reactors for Condensable Tar Production from Biomass, Energy \& Fuels 12(1), 25-40.

[34] Di Blasi, C., 1996. Heat, momentum and mass transport through a shrinking biomass particle exposed to thermal radiation, Chemical Engineering Science 51(7), 1121-1132. of solid particles undergoing fast pyrolysis, Chemical Engineering Science, 41(1), 151-157. Studies of the Effects of Volume Shrinkage on the Pyrolysis of Waste Wood Sphere, Energy \& Fuels 28(10), 6398-6406.

[37] Wang, Q., Zhao, Y. \& Zhang, Y., 2014. Shrinkage kinetics of large-sized briquettes during pyrolysis and its application in tamped coal cakes from large-scale chambers, Fuel 138, 1-14. moments to polydisperse gas-solid fluidized beds, Powder Technology 139(1), 7-20. distribution in CFD simulations of polymerization reactors, The 12th International Conference on Fluidization-New Horizons in Fluidization Engineering, 992-1000. [40] Marchisio, D.L. \& Fox, R.O., 2005. Solution of population balance equations using the direct quadrature method of moments, Journal of Aerosol Science 36(1), 43-73. moment method for dilute to moderately dilute fluid-particle flows, Chemical Engineering Science 65(7), 2267-2283.

877 [42] Gidaspow, D., 1994. Multiphase flow and fluidization: continuum and kinetic theory descriptions. 878 Academic press.

879 [43] Gidaspow, D., Bezburuah, R. \& Ding, J., 1991. Hydrodynamics of circulating fluidized beds: kinetic 
theory approach (No. CONF-920502-1). Illinois Inst. of Tech., Chicago, IL (United States). Dept. of Chemical Engineering.

882

[44] Syamlal, M., Rogers, W. \& O'Brien, T., 1993. MFIX Documentation: Volume 1, Theory Guide.

[45] Schaeffer, D.G., 1987. Instability in the evolution equations describing incompressible granular flow, Journal of Differential Equations 66(1), 19-50.

885

[46] Lun, C.K.K., Savage, S.B., Jeffrey, D.J. \& Chepurniy, N., 1984. Kinetic theories for granular flow: 886 inelastic particles in Couette flow and slightly inelastic particles in a general flowfield, Journal of Fluid Mechanics 140, 223-256.

888

[47] Syamlal, M., 1987. The Particle-Particle Drag Term in a Multiparticle Model of Fluidization, Eg\&G

Washington Analytical Services Center, Inc., Morgantown, WV(USA).

[48] Gunn, D.J., 1978. Transfer of heat or mass to particles in fixed and fluidised beds, International Journal of Heat and Mass Transfer 21(4), 467-476.

[49] Collier, A.P., Hayhurst, A.N., Richardson, J.L., \& Scott, S.A., 2004. The heat transfer coefficient between a particle and a bed (packed or fluidised) of much larger particles, Chemical Engineering Science 59(21), 4613-4620.

[50] Curtis, L.J. \& Miller, D.J., 1988. Transport model with radiative heat transfer for rapid cellulose pyrolysis, Industrial \& Engineering Chemistry Research 27(10), 1775-1783.

897

[51] Di Blasi, C., 1993. Analysis of Convection and Secondary Reaction Effects within Porous Solid Fuels undergoing Pyrolysis, Combustion Science and Technology 90(5-6), 315-340.

[52] Miller, R.S. \& Bellan, J., 1996. Analysis of Reaction Products and Conversion Time in the Pyrolysis of Cellulose and Wood Particles, Combustion Science and Technology 119(1-6), 331-373.

901 [53] Shafizadeh, F. \& Bradbury, A.G.W., 1979. Thermal Degradation of Cellulose in Air and Nitrogen At 902 Low Temperatures, Journal of Applied Polymer Science 23(5), 1431-1442.

903 [54] Ward, S.M. \& Braslaw, J., 1985. Experimental weight loss kinetics of wood pyrolysis under vacuum, 904 Combustion and Flame 61(3), 261-269. 
906

907

908

909

910

911

912

913

914

915

916

917

918

919

920

921

922

923

924

925

926

927

928

929

and biomass components, The Canadian Journal of Chemical Engineering 67(1), 75-84.

[56] Miller, R.S. \& Bellan, J., 1997. A Generalized Biomass Pyrolysis Model Based on Superimposed Cellulose, Hemicellulose and Lignin Kinetics, Combustion Science and Technology 126(1-6), 97-137. [57] Jin, W., Singh, K. \& Zondlo, J., 2013. Pyrolysis Kinetics of Physical Components of Wood and WoodPolymers Using Isoconversion Method, Agriculture 3(1), 12-32.

[58] Koufopanos, C.A., Papayannakos, N., Maschio, G. \& Lucchesi, A., 1991. Modelling of the Pyrolysis of Biomass Particles. Studies on Kinetics, Thermal and Heat Transfer Effects, The Canadian journal of chemical engineering 69(4), 907-915.

[59] Di Blasi, C., 1994. Numerical Simulation of Cellulose Pyrolysis, Biomass and Bioenergy 7(1), 87-98. [60] Randolph, A.D. \& Larson, M.A., 1971. Theory of Particulate Processes. New York: Academic Press. [61] McGraw, R., 1997. Description of Aerosol Dynamics by the Quadrature Method of Moments, Aerosol Science and Technology 27(2), 255-265.

[62] Fan, L.T., Fan, L.S., Miyanami, K., Chen, T.Y. \& Walawender, W.P., 1977. A Mathematical Model for Pyrolysis of a Solid Particle: Effects of the Lewis Number, The Canadian Journal of Chemical Engineering 55(1), 47-53.

[63] Min, J., Drake, J.B., Heindel, T.J. \& Fox, R.O., 2010. Experimental Validation of CFD Simulations of a Lab-Scale Fluidized-Bed Reactor with and without Side-Gas Injection, AIChE journal 56(6), 1434-1446. [64] Liu, D. \& Chen, X., 2010. Lateral Solids Dispersion Coefficient in Large-scale Fluidized Beds, Combustion and Flame 157(11), 2116-2124.

[65] Harlow, F.H. \& Welch, J.E., 1965. Numerical Calculation of Time - dependent Viscous Incompressible Flow of Fluid with Free Surface, The Physics of Fluids 8(12), 2182-2189.

[66] Yeung, P.K. \& Pope, S.B., 1988. An Algorithm for Tracking Fluid Particles in Numerical Simulations of Homogeneous Turbulence, Journal of Computational Physics, 79(2), 373-416.

[67] Anderson, M.P. \& Woessner, W.W., 1992. Applied Groundwater Modelling. Academic Press. 
930 [68] Genel, S., Vogelsberger, M., Nelson, D., Sijacki, D., Springel, V. \& Hernquist, L., 2013. Following 931 the Flow: Tracer Particles in Astrophysical Fluid Simulations, Monthly Notices of the Royal 932 Astronomical Society, 435(2), 1426-1442.

933 [69] Federrath, C., Glover, S.C.O., Klessen, R.S. \& Schmidt, W., 2008. Turbulent Mixing in the 934 Interstellar Medium: an Application for Lagrangian Tracer Particles. Physica Scripta, 2008(T132), 935014025.

936 [70] Vazza, F., Gheller, C. \& Brunetti, G., 2010. The Mixing and Transport Properties of the Intra Cluster 937 Medium: a Numerical Study Using Tracers Particles, Astronomy \& Astrophysics, 513, A32.

938 [71] Patel, M., 2013. Pyrolysis and gasification of biomass and acid hydrolysis residues (Doctoral 939 dissertation, Aston University).

940 [72] Di Blasi, C., 2008. Modeling chemical and physical processes of wood and biomass pyrolysis, 941 Progress in Energy and Combustion Science 34(1), 47-90.

942

943

944

945

946

947

948 
950 Appendix

951 Some of particle trajectories (10 trajectories generally in each diagram)



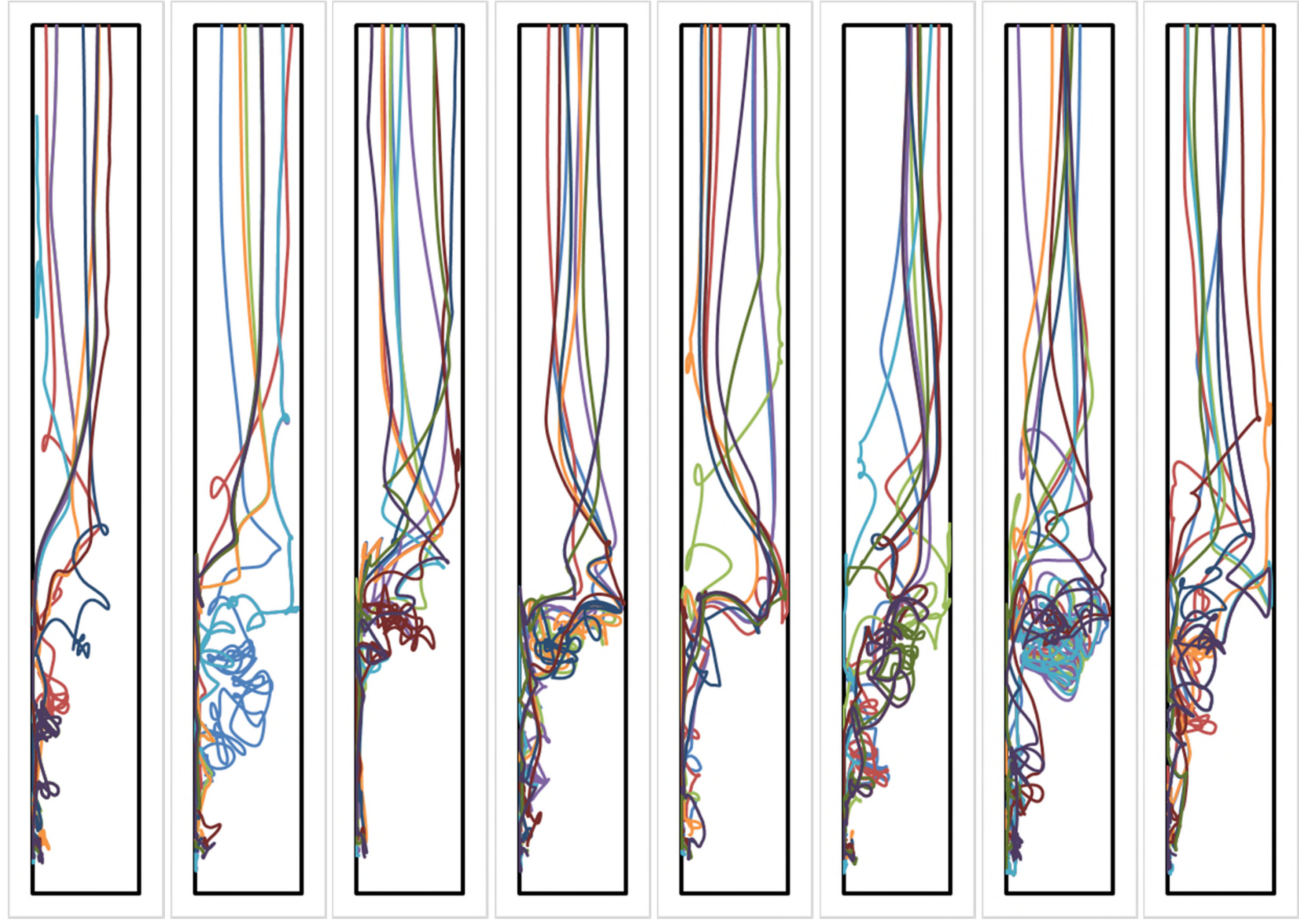

Fig. A.1 Particle trajectories for no-shrinkage pattern
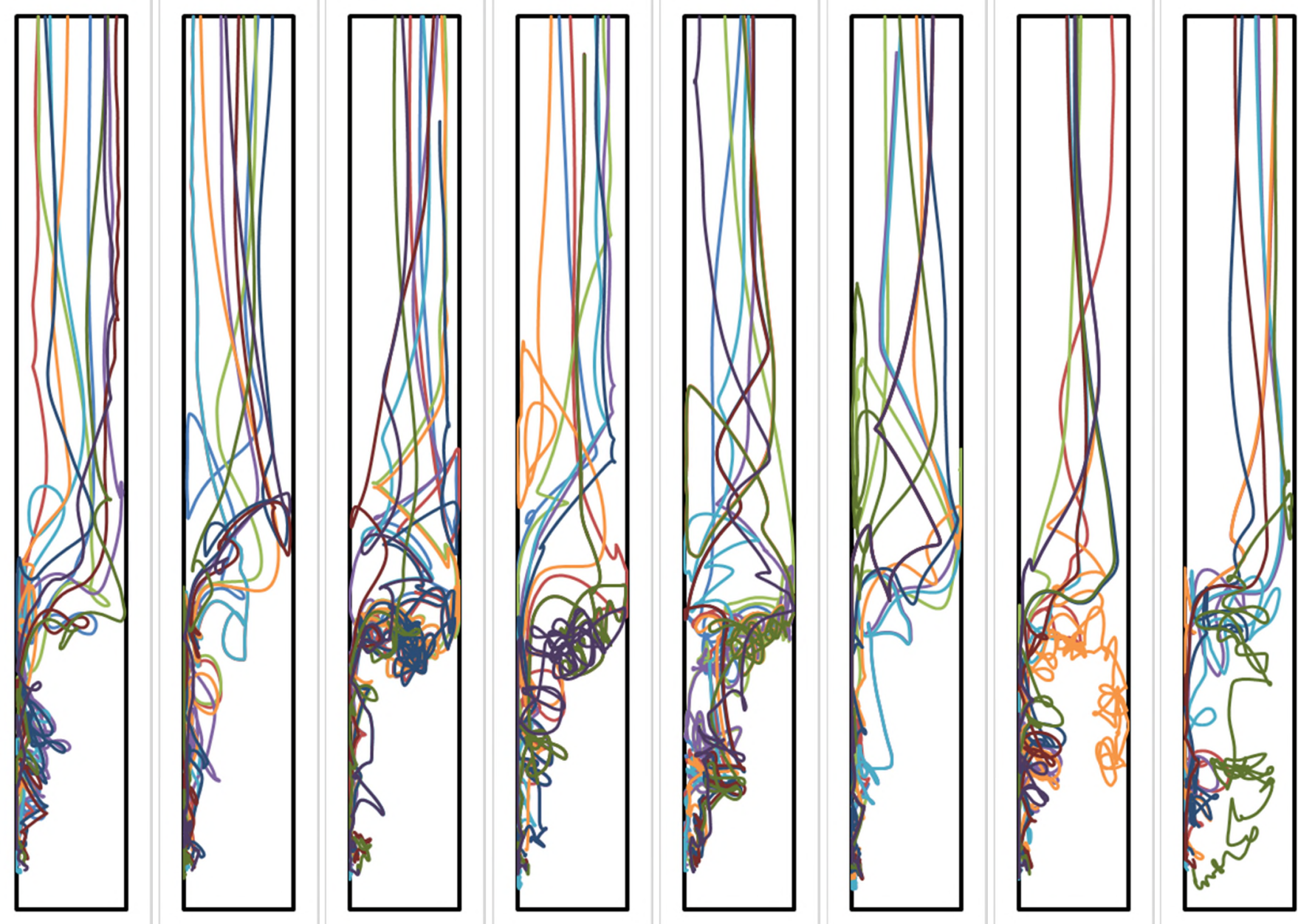


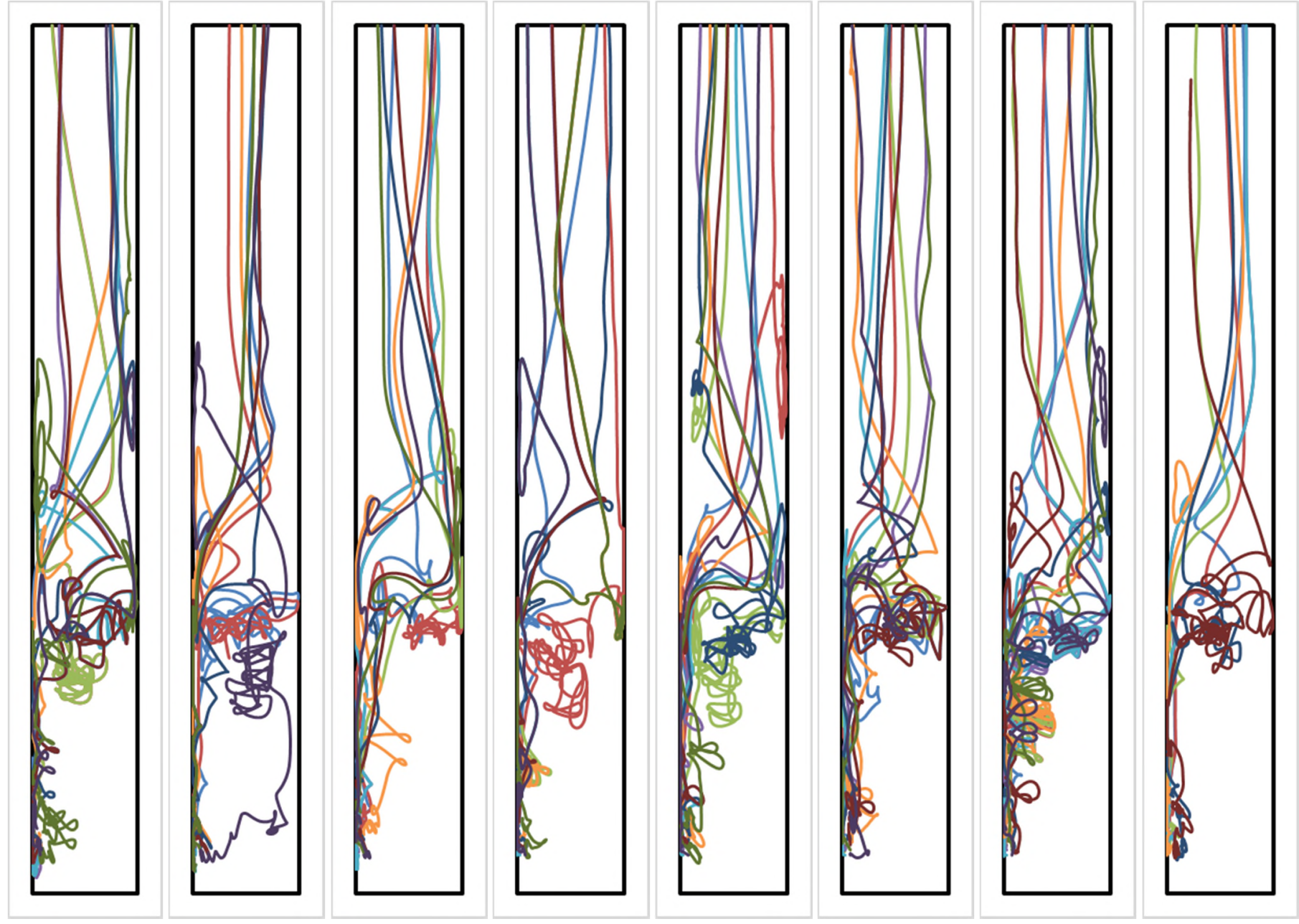

Fig. A.2 Particle trajectories for shrinkage pattern 1


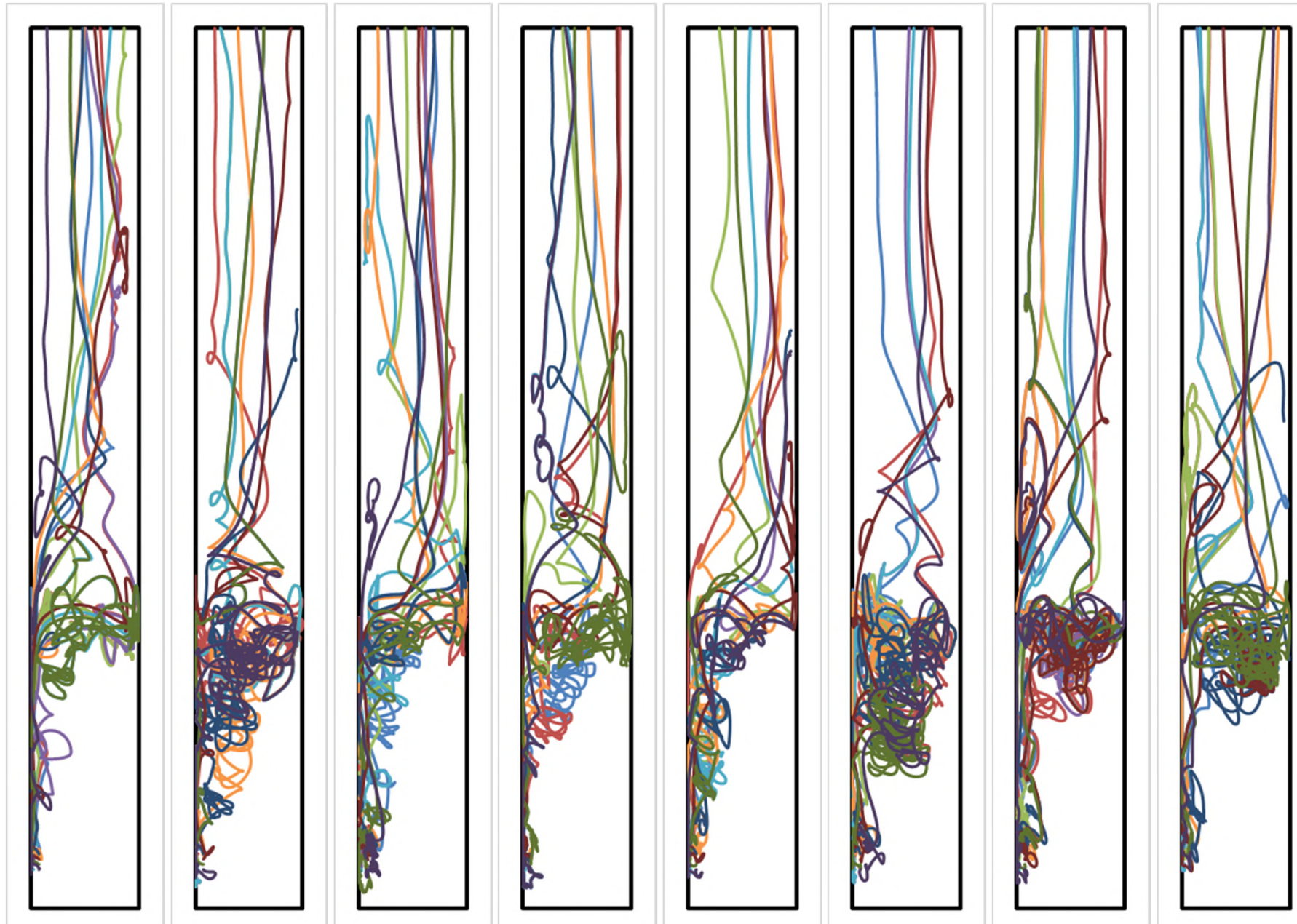

Fig A.3 Particle trajectories for shrinkage pattern 2 